\title{
KINETIC STUDIES OF ACID HYDROLYSIS OF FOOD WASTE-DERIVED SACCHARIDES
}

by

Elvis Osamudiamhen Ebikade

A thesis submitted to the Faculty of the University of Delaware in partial fulfillment of the requirements for the degree of Master of Chemical Engineering

2019 Fall

(C) 2019 Elvis Ebikade

All Rights Reserved 
KINETIC STUDIES OF ACID HYDROLYSIS OF FOOD WASTE-DERIVED

\section{SACCHARIDES}

by

Elvis Osamudiamhen Ebikade

Approved:

Dionisios Vlachos, Ph.D.

Professor in charge of thesis on behalf of the Advisory Committee

Approved:

Eric Furst, Ph.D.

Chair of the Department of Chemical and Biomolecular Engineering

Approved:

Levi T. Thompson, Ph.D.

Dean of the College of Engineering

Approved:

Douglas J. Doren, Ph.D.

Interim Vice Provost for Graduate \& Professional Education and Dean of the Graduate College 


\section{ACKNOWLEDGMENTS}

To God - the giver of life, and the source of all wisdom, I ascribe my sincere thanks and gratitude.

To my advisor - Professor Dionisios Vlachos, thank you for your patient guidance, feedback and valuable mentorship during my research.

This work was supported as part of the Catalysis Center for Energy Innovation, an Energy Frontier Research Center funded by the U.S. Department of Energy, Office of Science, and Office of Basic Energy Sciences under award number DE-SC0001004. I also acknowledge support from the Allan and Myra Ferguson Fellowship.

Thank you to every member of the Vlachos lab, especially Natalia, Yifan and Gerhard for their support and assistance during my graduate career. Special thanks to Gerhard Wittreich, Jonathan Lym and Dr. Basudeb Saha who worked with me on this project.

Finally, sincere appreciation to my parents and family members, who constantly supported me with their prayers and calls. 


\section{TABLE OF CONTENTS}

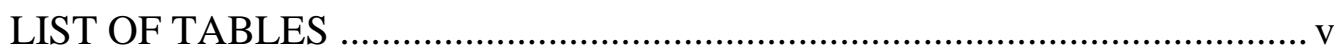

LIST OF FIGURES ………………………………................................ vi

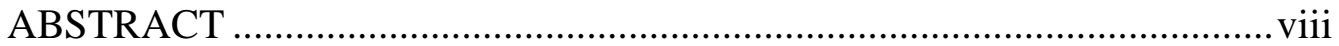

Chapter

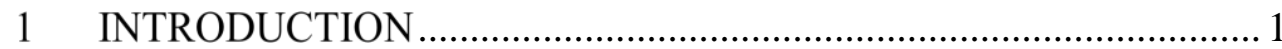

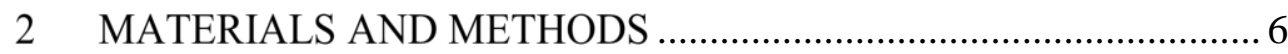

2.1 Experimental materials and methods............................................... 6

2.2 Mathematical modeling and computational methods .................... 10

3 RESULTS AND DISCUSSIONS ………………………………....... 13

3.1 Hydrolysis of malto-oligosaccharides ........................................... 13

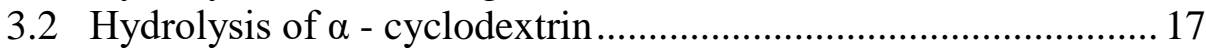

3.3 Hydrolysis of starch................................................................... 19

3.4 Effect of saccharide chain length on hydrolysis ............................2 23

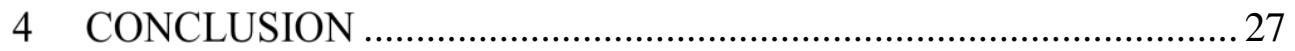

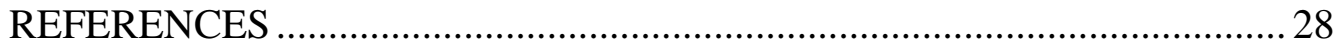

Appendix

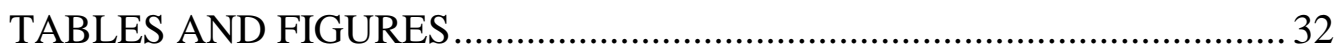




\section{LIST OF TABLES}

Table 1.1: Ratio of bond scission rate constants for the acid-catalyzed hydrolysis of various saccharides...................................................................... 3

Table 3.1: Rate constants for hydrolysis of glycosidic bonds of saccharides. ............ 14

Table A.1: Comparison of maximum glucose yields in different hydrolysis medium 32

Table A.2: Rate constants $\mathrm{k}_{1}$ and $\mathrm{k}_{2}$ at different temperatures .............................. 32 


\section{LIST OF FIGURES}

Figure 2.1: Glucan unit and bond nomenclature........................................................ 10

Figure 2.2: Hydrolysis pathways of a pentasaccharide... ............................................. 11

Figure 3.1: Hydrolysis mechanism of malto-saccharides............................................. 13

Figure 3.2: Time profiles of products from (a) maltose hydrolysis and (b) maltotriose hydrolysis in $\mathrm{LiBr}$ AMSH............................................................... 15

Figure 3.3: Time profile of maltotetraose hydrolysis in LiBr AMSH........................... 17

Figure 3.4: Time profile of $\alpha$ - cyclodextrin hydrolysis in LiBr AMSH....................... 19

Figure 3.5: X-ray diffraction pattern of potato and gelatinised starch. .........................20

Figure 3.6: Time profile of glucose concentration from potato starch hydrolysis (amorphous + crystalline) in LiBr AMSH..............................................21

Figure 3.7: Time profile of products from potato starch hydrolysis in LiBr AMSH... 22

Figure 3.8: Effect of chain length on (a) saccharide hydrolysis rate constant and (b) glucose formation rate constant..............................................................24

Figure 3.9: Simulated glucose formation profile from hydrolysis of oligosaccharides or polysaccharides of different chain length................................................25

Figure A.1: Parity plot of predicted vs. experimental data from maltotetraose

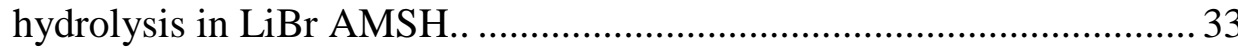

Figure A.2: Predicted vs. experimental data from $\alpha$-cyclodextrin hydrolysis in $\mathrm{LiBr}$ AMSH for all reaction products............................................................. 34

Figure A.3: Time dependent profile of observable intermediates from $\alpha$-cyclodextrin hydrolysis in $\mathrm{LiBr}$ AMSH.

Figure A.4: Particle size distribution of potato starch in LiBr MSH (before hydrolysis)

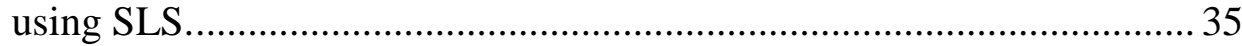


Figure A.5: Particle size distribution of potato starch in LiBr AMSH after hydrolysis

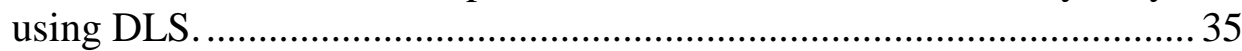

Figure A.6: Parity plot of predicted vs. experimental concentrations of products from potato starch hydrolysis in $\mathrm{LiBr}$ AMSH.............................................36

Figure A.7: Hydrolysis profile of oligosaccharides with different chain length.......... 36

Figure A.8: Comparison of glucose yield profile from oligosaccharides with different

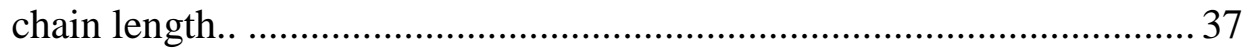




\begin{abstract}
Starch saccharified glucose from food waste can be an important precursor for renewable chemicals and fuels. Despite numerous studies on hydrolysis of biomass, detailed kinetic studies and associated models of hydrolysis are lacking. We investigated the kinetics of glycosidic bond scission of malto-oligosaccharides in lithium bromide acidified molten salt hydrate (AMSH) medium and estimated rate parameters from experimental data. Our data supports the hypothesis that the terminal, non-reducing bonds hydrolyze faster than the interior and terminal-reducing $\mathrm{C}-\mathrm{O}$ bonds. Next, we extended the model to simulate the hydrolysis of linear and cyclic saccharides of varying degree of polymerization and of potato starch. We characterize starch using $\mathrm{X}$-ray diffraction (XRD) and light scattering methods. The model is in excellent agreement with the experimentally determined concentrations of glucose and other oligosaccharides. The chain length of saccharides is found to be directly related to their hydrolysis rate constant, but inversely proportional to the glucose formation rate constant.
\end{abstract}




\section{Chapter 1 INTRODUCTION}

Non-food biomass deconstruction and valorization of fractionated carbohydrates into high value chemicals and fuels have received significant attention over the past decade ${ }^{1,2}$. Cellulose and starch are natural polysaccharides consisting of glucose, which is a precursor for manufacturing some of the top-ten bio-based chemicals listed by the United States Department of Energy ${ }^{3}$. As a result, energy-efficient hydrolysis of polysaccharides to glucose has been investigated by several groups ${ }^{1,4-12}$.

Polysaccharide hydrolysis kinetics was first described by Saeman et al. ${ }^{13}$ They developed an empirical description for cellulose hydrolysis where the first reaction involves the conversion of cellulose into the reducing moiety - glucose, and the second reaction involves glucose conversion to other products, such as 5-hydroxymethyl furfural (HMF), levulinic acid (LA), formic acid (FA) etc., which they referred to as degradation products

$$
\text { Cellulose } \rightarrow \text { reducing sugar } \rightarrow \text { sugar degradation products }
$$

Additional studies, based on Saeman's empirical model, have been carried out by Fagan $e$ a al. ${ }^{14}$ and others ${ }^{15-20}$. Glucose units in cellulose are bonded by $\beta(1-4)$ glycosidic linkages, which are oriented equatorially unlike the axially oriented $\alpha(1-4)$ glycosidic linkages between D-glucopyranose units in starch. Aside the contributions of inter- and intra-molecular hydrogen bond network in cellulose, the difference in bond orientation also contributes to the distinct hydrolysis reactivity of starch and cellulose. 
Food waste presents an attractive, non-conventional, alternative feedstock yet to be explored for producing renewable fuels and chemicals. Its high carbohydrate content, i.e., $33-74 \%{ }^{12,21}$ on a dry weight is appealing. For example, the EU generates 8 million metric tons of starch waste annually ${ }^{21}$. This provides six times the required amount of glucose needed to meet the global demand for lactic acid, succinic acid, fumaric acid and polyhydroxybutyrate (PHB). ${ }^{21}$ Despite the large number of products that can be obtained from valorization of starch-rich food waste, the number of kinetic studies of starch (malto-polysaccharide) hydrolysis is very limited compared to cellulose hydrolysis. Given the growing interest in valorization of starch-rich food waste ${ }^{12,21-23}$ (e.g. potato peel, bread waste, etc.), a detailed kinetic study for the scission of $\alpha-$ glycosidic bonds in starch would be valuable.

Various hypotheses for the hydrolysis of inter-unit C-O linkages in linear saccharides have been reported ${ }^{24-31}$. These include: (1) Bonds at the reducing and nonreducing terminal ends are hydrolyzed at a higher rate than all other bonds. (2) The hydrolysis of all bonds occurs at the same rate, irrespective of their position in the chain and the degree of polymerization (DP) of the chain. (3) The bond at the non-reducing end is hydrolyzed faster than all other bonds, while all other bonds are hydrolyzed at the same rate. (4) There is a progressive decrease in the hydrolysis rate constants from the terminal bond towards the interior bonds. While prior research by Freundeberg et $a l^{32}$ and Nguyen et $a l^{33}$ support hypotheses 1 and 4, hypothesis 3 is the most widely accepted hypothesis and supported by several experimental results ${ }^{25-31,34}$ and is the one exploited in this study. An overview of kinetic studies for cello-, malto- or xylosaccharides and the ratio of hydrolysis rates of the non-reducing terminal bond to that of all other bonds within the chain are given in Table 1.1. 
Table 1.1: Ratio of bond scission rate constants for the acid-catalyzed hydrolysis of various saccharides.

\begin{tabular}{lrll}
\hline Substrate & $\mathrm{k}_{1} / \mathrm{k}_{2}$ & Reaction conditions & Reference \\
\hline Cello-oligosaccharide & 1.7 & $\mathrm{~T}: 90-135^{\circ} \mathrm{C}$ \\
& & $\mathrm{C}_{\text {substrate }}: 0.5-1 \mathrm{wt} . \%$ \\
& & $\mathrm{C}_{\mathrm{H} 2 \mathrm{SO} 4}: 0.05-0.1 \mathrm{~N}$ \\
\hline Cello-oligosaccharide & 1.5 & $\mathrm{~T}: 30-120^{\circ} \mathrm{C}$ \\
& & $\mathrm{C}_{\text {substrate }}: 10 \mathrm{wt} \%$ \\
& & $\mathrm{C}_{\mathrm{H} 2 \mathrm{SO} 4}: 0.5-14.2 \mathrm{~N}$ \\
\hline Malto-oligosaccharide & 1.8 & $\mathrm{~T}: 95{ }^{\circ} \mathrm{C}$ \\
& & $\mathrm{C}_{\text {substrate }}: 0.1-0.4 \mathrm{wt} . \%$ \\
& & $\mathrm{C}_{\mathrm{H} 2 \mathrm{SO} 4}: 0.01 \mathrm{~N}$ \\
\hline Malto-oligosaccharide & 1.8 & $\mathrm{~T}: 75-95{ }^{\circ} \mathrm{C}$ \\
& & $\mathrm{C}_{\text {substrate }}: 0.625 \mathrm{wt} \%$ \\
& & $\mathrm{C}_{\mathrm{H} 2 \mathrm{SO} 4}: 0.01 \mathrm{~N}$ \\
\hline Malto-oligosaccharide & 1.7 & $\mathrm{~T}: 70-135{ }^{\circ} \mathrm{C}$ \\
& & $\mathrm{C}_{\text {substrate }}: 1 \mathrm{wt} . \%$ \\
& & $\mathrm{C}_{\mathrm{H} 2 \mathrm{SO} 4}: 0.2 \mathrm{~N}$ \\
\hline Xylo-oligosaccharide & 1.7 & $\mathrm{~T}: 80-100{ }^{\circ} \mathrm{C}$ \\
& & $\mathrm{C}_{\text {substrate }}: 1 \mathrm{wt} \%$ \\
& & $\mathrm{C}_{\mathrm{H} 2 \mathrm{SO} 4}: 0.05-0.5 \mathrm{~N}$ \\
\hline Xylo-polysaccharide & 1.3 & $\mathrm{~T}: 80{ }^{\circ} \mathrm{C}$ \\
& & $\mathrm{C}_{\text {substrate }}: 5.4 \mathrm{wt} . \%$ in $[\mathrm{Emim}][\mathrm{Cl}]$ \\
& $\mathrm{C}_{\mathrm{H} 2 \mathrm{SO} 4}: 0.4 \mathrm{~N}$ \\
\hline
\end{tabular}

$\mathrm{k}_{1}=$ rate constant for $\mathrm{C}-\mathrm{O}$ bonds scission at non-reducing terminal; $\mathrm{k}_{2}=$ rate constant for $\mathrm{C}-\mathrm{O}$ bonds scission at reducing terminal and interior linkages

As shown in Table 1.1, the pseudo-first-order hydrolysis rate constant $\left(\mathrm{k}_{1}\right)$ for C-O bonds scission of various saccharides at the non-reducing end is $1.3-1.8$ times higher than that of reducing end and interior bonds $\left(\mathrm{k}_{2}\right)$. While these kinetic studies have been carried out in popular solvents (conventional acid and ionic liquids) for saccharides' hydrolysis, no kinetic study has been carried out on the bond scission of saccharides in AMSH. 
Recently, acidified molten salt hydrates (AMSHs) mediated hydrolysis of cellulose and lignocellulosic biomass have been reported ${ }^{4,5,8,35-38}$. Lithium bromide based MSH (59 wt.\% LiBr solution in water at water to $\mathrm{LiBr}$ molar ratio of 3.25) saccharified crystalline cellulose $e^{4,5,38}$ to soluble sugars with a very high yield (>90\%) containing glucose as the major product $(82 \%)$ at low temperature $\left(85^{\circ} \mathrm{C}\right)$ and short reaction time (30 min). It has been reported that AMSH becomes super-acidic via polarization of hydrogen of water in the hydration sphere of the cation and a synergy between the salt and added acid ${ }^{4,35,39}$, facilitating hydrolysis at faster rates with minimal degradation products (e.g. HMF, humins etc.). LiBr Molten-salt alone is not acidic ( $\mathrm{pH}$ $\sim 6)^{4}$. While AMSH has been used for cellulose hydrolysis ${ }^{5,38}$, its effectiveness for starch (an important component of food-waste) hydrolysis and related kinetic investigation has not been reported. This study will provide such information, necessary toward developing a food-waste based integrated biorefinery.

Importantly, strongly acidic AMSH is less corrosive $e^{4,37,38,40}$ in comparison with a pure acid of equivalent dissociated proton concentration (based on electrode potential measurement). In addition, AMSHs are easy to prepare, have low volatility and are cheaper than most ionic liquids ${ }^{5,36}$ which have been employed for hydrolysis. AMSHs also have good recyclability ${ }^{4,5}$, achieving high conversions and yields even after three cycles of use. The application of AMSH for starch has though remained elusive. Yan et al. ${ }^{41}$ achieved maximum glucose yields of 19\% (34\% selectivity) after $40 \mathrm{~min}$ of reaction at $160{ }^{\circ} \mathrm{C}$ using 1 wt.\% (0.2 M) $\mathrm{H}_{2} \mathrm{SO}_{4}$. By comparison, the LiBr AMSH efficiently dissolved and saccharified cellulose in one step giving $>90 \%$ yield of soluble sugars ${ }^{5,38}$ (82\% glucose selectivity) within $40 \mathrm{~min}$ at 85 ${ }^{\circ} \mathrm{C}$. Sadula et al. ${ }^{5}$ reported a lower minimum price of $\mathrm{HMF}$ manufactured using $\mathrm{LiBr}$ 
AMSH compared to concentrated and dilute acid systems. This cost advantage ${ }^{5}$ in $\mathrm{HMF}$ production using AMSHs is attributed to the single-stage conversion technology as well as the higher glucose yield (Table A.1).

In this study, we develop a fundamental, mass action kinetics model for hydrolysis of malto-oligosaccharides and polysaccharides (e.g., starch) in AMSH media that that can accurately predict species concentrations formed during hydrolysis. We show that two fundamental rate constants obtained from model compounds, maltose and maltotriose, are sufficient to model more complex macromolecules. We demonstrate that the model-predicted concentrations against time agree well with the experimentally determined concentrations of glucose, maltose, maltotriose and maltotetraose. The effect of chain length of saccharides on their hydrolysis rates and glucose yield is also discussed. 


\section{Chapter 2}

\section{MATERIALS AND METHODS}

\subsection{Experimental materials and methods}

Materials. Reagent grade maltose, $\alpha$-cyclodextrin, dextran (DP - 10), lithium bromide, $5 \mathrm{M}$ sulfuric acid and potato starch, were purchased from Sigma-Aldrich. Malto-oligosaccharides (maltotriose and maltotetraose) of $>95 \%$ purity were purchased from MegaZyme. All chemicals were used as received. Deionized water (Millipore model Direct Q3 UV R) was used for the preparation of all solutions. Syringe filter discs (Nylon, $0.2 \mu \mathrm{m}$ ) for the filtration of samples prior to HPLC analysis were purchased from Fisher Scientific.

Reactions. $\mathrm{LiBr}$ MSH solutions (59 wt.\% LiBr; water to LiBr molar ratio 3.25) were prepared by dissolving $\mathrm{LiBr}$ in deionized water and were equilibrated overnight at $60{ }^{\circ} \mathrm{C}$. For kinetic studies, the desired amount of starch or oligosaccharides was added into a $25 \mathrm{~mL}$ three-neck round-bottom flask containing $3.64 \mathrm{~mL} \mathrm{LiBr} \mathrm{MSH}$ solution. The mixture was heated to and equilibrated at desired reaction temperature on an IKA C-MAG HS hotplate stirrer, followed by addition of $25 \mu \mathrm{l}$ of $5 \mathrm{M}$ sulphuric acid to make the AMSH. The reaction mixture was continuously stirred at $800 \mathrm{rpm}$. Typical reaction conditions are $60{ }^{\circ} \mathrm{C}$, salt/glucan (glucose equivalent) ratio of 69 , and final sulphuric acid concentration of $0.05 \mathrm{M}$. A pipette was used to collect $\sim 0.1 \mathrm{~mL}$ of aliquot at regular time intervals that were immediately quenched in deionized water, diluted 20-times, and then filtered through a $0.2 \mu \mathrm{m}$ syringe filter disk to remove any unreacted solids before analysis by HPLC. 
Analysis and quantification of hydrolysis products. Product solutions were analyzed using a Waters e2695 HPLC separation unit equipped with photodiode array (PDA) (Waters 2998) and refractive index (RI) (Waters 2414) detectors. An Aminex Bio-Rad HPX-87H $(7.8 \times 300 \mathrm{~mm} \times 9 \mu \mathrm{m})$ column with preset column temperature of $55^{\circ} \mathrm{C}$ and a $0.005 \mathrm{M} \mathrm{H}_{2} \mathrm{SO}_{4}$ solution as a mobile phase (flow rate $=0.6 \mathrm{~mL} / \mathrm{min}$ ) were used to analyze to quantify the following products: maltose, glucose, and HMF. Maltooligosaccharides of DP $=3-5$ were quantified using an Agilent HiplexNa column (7.7 $\times 300 \mathrm{~mm} \times 10 \mu \mathrm{m})$ at $85^{\circ} \mathrm{C}$ using deionized water as a mobile phase with a flow rate of $0.2 \mathrm{~mL} / \mathrm{min}$. The characteristic peaks for sugar monosaccharides and oligosaccharides were identified from the retention times of the authentic samples. Each peak was integrated, and the actual concentration of each product was calculated from calibration plots.

\section{Rate constants.}

Initial hydrolysis and dehydration rate constants. The concentrations of all species during hydrolysis were determined using HPLC as described above. When the natural logarithm of the ratio of concentration of hydrolyzed species to the initial oligosaccharide concentration was plotted against time using Equation 2-1, a straight line was obtained for each set of reaction conditions, indicating the hydrolysis follows first-order kinetics in acid and substrate concentration $2,26,28,29,31,42$

$$
\ln \frac{C}{C_{o}}=-k t
$$

Here $c_{o}$ is the initial concentration of oligosaccharide $\left(\mathrm{mol} / \mathrm{dm}^{3}\right), c$ is the concentration of oligosaccharide at time $\mathrm{t}\left(\mathrm{mol} / \mathrm{dm}^{3}\right)$, and $\mathrm{k}$ is the apparent reaction rate constant $\left(\mathrm{min}^{-1}\right)$. 
Rate constant for $\mathrm{C}-\mathrm{O}$ bonds scission at non-reducing terminal $\left(\mathrm{k}_{1}\right)$ is obtained directly from maltose hydrolysis rate constant, while rate constant for $\mathrm{C}-\mathrm{O}$ bonds scission at reducing terminal and interior linkages $\left(\mathrm{k}_{1}\right)$ is obtained by subtracting maltotriose hydrolysis rate constant from the maltose hydrolysis rate constant, $\mathrm{k}_{1}$ as described in Equation 2-2.

$$
k_{2}=k_{\text {maltotriose }}-k_{\text {maltose }}
$$

The dehydration rate constant was determined from independent dehydration experiments starting from glucose in $\mathrm{LiBr} \mathrm{AMSH}$.

Glucose production rate constant. The rate constant of glucose formation from polysaccharides and oligosaccharides was determined from the slope of glucose fraction vs. time. The glucose fraction (Equation 2-3) varies between 0 and 1

$$
\text { Glucose fraction }=\frac{C_{\text {glucose }}}{C_{\text {oligosaccharide }, 0}}
$$

Here, $\mathrm{c}_{\text {glucose }}$ is the concentration of glucose in solution determined from HPLC, Coligosaccharide, 0 is the initial concentration of glucose in oligosaccharides, determined using the relation in Equation 2-4

$$
c_{\text {oligosaccharide }, 0}=\frac{m_{\text {oligosaccharide }} \times g_{\text {theoretical }}}{M W_{\text {glucose }} \times \text { vol }_{\text {reaction }}}
$$

$\mathrm{m}_{\text {oligosaccharide }}$ is the mass of oligosaccharide, $\mathrm{g}_{\text {theoretical }}$ is the theoretical glucose amount wt.\% in oligosaccharide, $\mathrm{MW}_{\text {glucose }}$ is the molecular weight of glucose, vol ${ }_{\text {reaction }}$ is the volume of the reaction mixture.

All initial rate constants were estimated for low $(<15 \%)$ conversion. For starch, since we cannot directly measure conversion, we measure its conversion indirectly from glucose yields (carbon balance is greater than 95\%). The rate constants reported are all apparent rate constants. This apparent rate constant is the product of the actual rate constant and the proton activity (Equations 2-5 \& 2-6) 


$$
\begin{aligned}
k_{\text {apparent }} & =k_{\text {actual }}\left[a_{H^{+}}\right] \\
a_{H^{+}} & =\gamma\left[C_{H^{+}}\right]
\end{aligned}
$$

Here $\gamma$ is the activity coefficient of hydrolysis solution, $C_{H^{+}}$is the proton concentration in solution, and $a_{H^{+}}$is the proton activity in solution. Proton activity was measured using a $\mathrm{pH}$ meter. To address the non-ideality of AMSH, further work is needed that will be reported in a separate manuscript.

Temperature effects. Rate constants $\left(\mathrm{k}_{1}\right.$ and $\left.\mathrm{k}_{2}\right)$ were obtained at temperatures of 40,50 and $60{ }^{\circ} \mathrm{C}$. These values were then fit to an Arrhenius plot to obtain the preexponential factor $(A)$ and apparent activation energy $\left(E_{a}\right)$.

pH measurements. The $\mathrm{pH}$ of the $\mathrm{LiBr}$ AMSH solution was measured using a pH meter (Fisher Scientific accumet Basic AB15). The electrode was calibrated with standard solutions of appropriate $\mathrm{pH}$ values before each measurement. Each solution $\mathrm{pH}$ was measured in triplicate.

X-ray powder diffraction (XRD) characterization. The XRD patterns were collected at room temperature using a Bruker D8 Discover X-Ray diffractometer using $\mathrm{Cu} \mathrm{K} \alpha$ radiation $(\lambda=1.5418 \AA)$ source. The data were collected in a stepwise fashion of $2 \theta$ ranging from $5^{\circ}$ to $60^{\circ}$ and a step size of $0.05^{\circ}$ and $1 \mathrm{~s} \mathrm{per} \mathrm{step.} \mathrm{Potato} \mathrm{starch} \mathrm{was}$ used as purchased for XRD measurements.

Starch gelatinization. Potato starch was heated in water for $30 \mathrm{~min}$ at $80^{\circ} \mathrm{C}$. This was carried out to provide direct evidence of the conversion of crystalline regions in potato starch to amorphous material. The resulting solution was freezed with liquid nitrogen and dried in a freeze dryer.

Dynamic light scattering (DLS). DLS experiments were conducted on a Brookhaven ZETAPALS instrument. DLS measurements were obtained for background 
blank solutions and starch hydrolysate. The autocorrelation functions were analyzed using the Multimodal Size Distribution algorithm of the accompanying software.

Static light scattering (SLS). SLS experiments were conducted on a Beckman Coulter LS 13320 Particle Size Analyzer instrument. Signals were analyzed using the Fraunhofer theory of light scattering. Particle obscuration was higher than $8 \%$ before the analysis was carried out.

\subsection{Mathematical modeling and computational methods}

Physical model

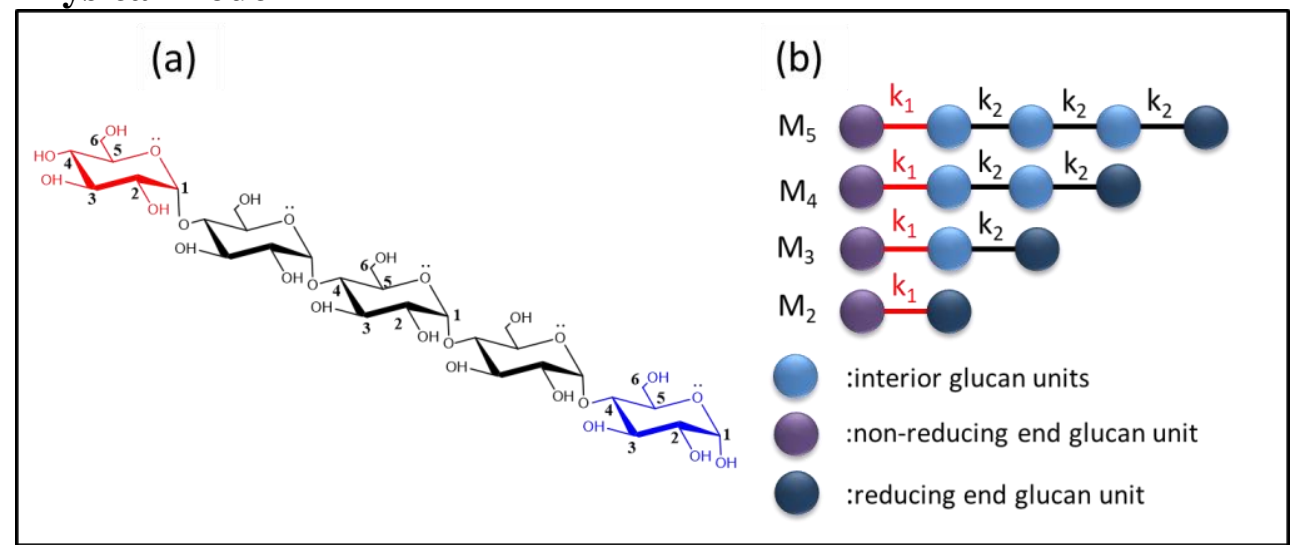

Figure 2.1: Glucan unit and bond nomenclature. (a) The reducing end (colored blue) has the anomeric carbon, $\mathrm{C} 1$ with the hemiacetal unit not involved in a $\mathrm{C}-\mathrm{O}$ linkage. The non-reducing end (colored red) has $\mathrm{C} 4$ not involved in a $\mathrm{C}-\mathrm{O}$ linkage. The interior units have both $\mathrm{C} 1$ and $\mathrm{C} 4$ involved in a $\mathrm{C}-\mathrm{O}$ linkage. (b) Schematic showing non-reducing end bond (with rate constant $\mathrm{k}_{1}$ ) and other bonds (with rate constant $\mathrm{k}_{2}$ ) in select oligosaccharides.

Figure 2.1 illustrates non-reducing terminal bonds with hydrolysis rate constant of $\mathrm{k}_{1}$ and other interior or reducing terminal bonds with hydrolysis rate constant of $\mathrm{k}_{2}$ 
for all linear saccharides. Glycosidic bond protonation and scission occur randomly in a chain. Consistent with this hypothesis, Joksimovic et al. ${ }^{43}$ used the PM3 molecularorbital method to identify random scission of glycosidic bonds during acid hydrolysis of carbohydrates. For illustration, the paths of hydrolysis are described for a pentasaccharide $\left(\mathrm{M}_{5}\right)$ in Figure 2.2. Hydrolysis products, tetrasaccharide $\left(\mathrm{M}_{4}\right)$, trisaccharide $\left(\mathrm{M}_{3}\right)$, disaccharide $\left(\mathrm{M}_{2}\right)$, and monosaccharide $\left(\mathrm{M}_{1}\right)$, are also shown in the scheme.
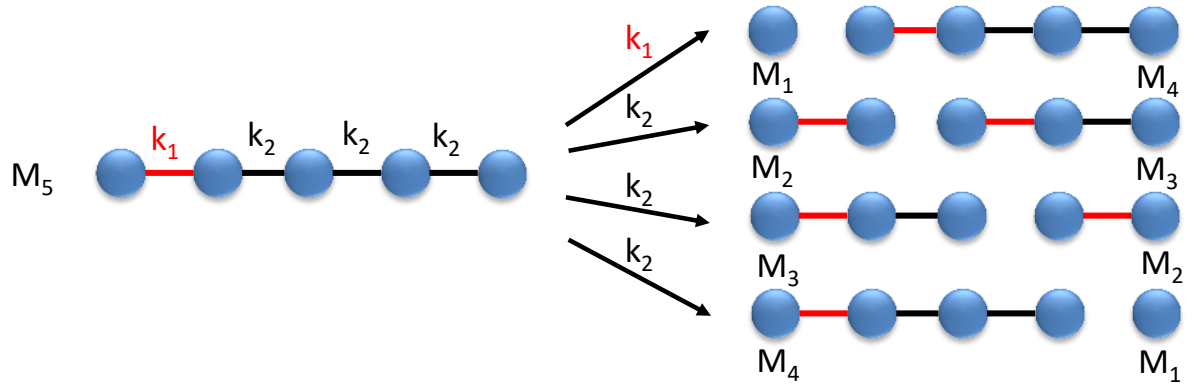

: glucan unit

Figure 2.2: Hydrolysis pathways of a pentasaccharide. A saccharide of $(m+n)$ units hydrolyzes into two species of $m$ and $n$ units each. The model (Equations 2-7 - 2-9) describes all $(m+n)$ species.

\subsection{Governing equations}

The following rate equations were used to describe the hydrolysis of a saccharide containing $\mathrm{n}$ - number of glucose units

The consumption rate of an $\mathrm{n}$-saccharide $(\mathrm{n}>1)$ is

$$
\frac{d C_{n}}{d t}=-\left[k_{1}+(n-2) k_{2}\right] C_{n}
$$

The rate of intermediate formation ( $\mathrm{i}>1)$ is

$$
\frac{d c_{i}}{d t}=\left(k_{1}+k_{2}\right) C_{i+1}+\sum_{j=i+2}^{n} 2 k_{2} C_{j}
$$


The rate of glucose formation is

$\frac{d C_{g}}{d t}=2 k_{1} C_{2}+\sum_{j=3}^{n}\left(k_{1}+k_{2}\right) C_{j}-k_{d} C_{g}$

Here $\mathrm{k}_{1}$ is the fast bond scission rate $\left(\min ^{-1}\right.$ per bond); $\mathrm{k}_{2}$ is the slow bond scission rate $\left(\mathrm{min}^{-1}\right.$ per bond); $\mathrm{k}_{\mathrm{d}}$ is the glucose dehydration rate $\left(\mathrm{min}^{-1}\right) ; \mathrm{c}_{\mathrm{n}}$ is the initial saccharide concentration $\left(\mathrm{mol} / \mathrm{dm}^{3}\right) . \quad \mathrm{c}_{\mathrm{i} / \mathrm{j}}$ is the intermediate oligosaccharide concentration $\left(\mathrm{mol} / \mathrm{dm}^{3}\right) . \mathrm{c}_{2}$ is the maltose concentration $\left(\mathrm{mol} / \mathrm{dm}^{3}\right) . \mathrm{c}_{\mathrm{g}}$ is the glucose concentration $\left(\mathrm{mol} / \mathrm{dm}^{3}\right)$. The derivation of rate equations and the solution of ODEs for the series reactions involving maltotriose and maltose hydrolysis is in the supporting information. The solution of the equations was carried out using the MATLAB® software, version R2017a. 


\section{Chapter 3}

\section{RESULTS AND DISCUSSIONS}

\subsection{Hydrolysis of malto-oligosaccharides}

Saccharide
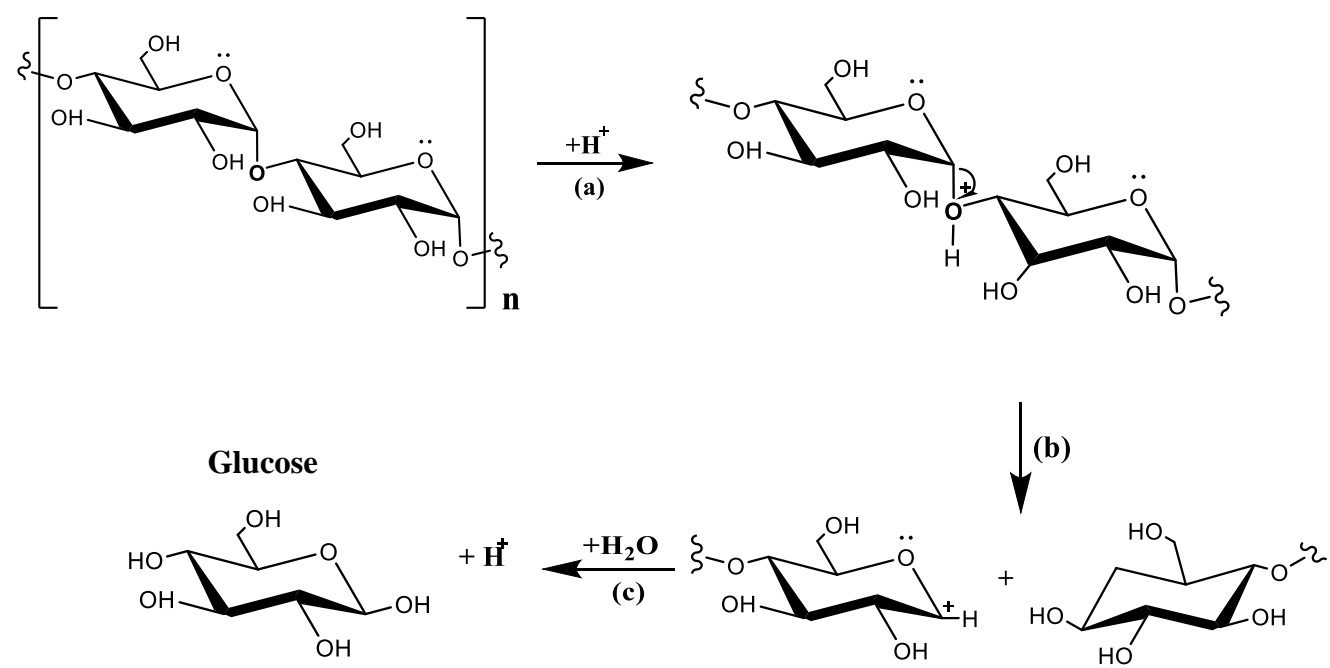

Figure 3.1: Hydrolysis mechanism of malto-saccharides.

The hydrolysis of any malto-saccharide is shown in Figure 3.1. The reaction ${ }^{24}$ is initiated by protonation (a) of a glycosidic oxygen connecting two adjacent glucan units. This follows heterolysis (b) of the protonated glycosidic bond to form a cyclic oxonium carbenium ion, and nucleophilic addition of water (c) to the carbenium ion to form glucose and release a proton. Hydrolysis of any saccharide of high DP substrates yields shorter chain species, which ultimately get converted to glucose. Under strong acidic conditions $^{4,5,38}$, glucose can further be dehydrated into HMF.

The time-profiles of maltose and maltotriose hydrolysis are illustrated in Figure 1. The two rate constants, $\mathrm{k}_{1}$ and $\mathrm{k}_{2}$, can be estimated using these substrates since they contain a non-reducing end and other glycosidic bonds. The disappearance of both 
substrates follows first-order kinetics. The hydrolysis profile of maltotriose (Figure 3.2b) shows formation of maltose as an intermediate which is subsequently hydrolyzed to glucose (Figures 3.2a and 3.2b). Upon regression, the model describes the experimental data reasonably. The estimated rate constants (see methods) are shown in Table 3.1.

Table B: Rate constants for hydrolysis of glycosidic bonds of saccharides.

Non- reducing end bond, $\mathrm{k}_{1} \quad$ Reducing end \& interior bonds, $\mathrm{k}_{2}$

\begin{tabular}{ccc}
$\left(\mathrm{min}^{-1}\right)$ & $\left(\mathrm{min}^{-1}\right)$ & $\mathrm{k}_{\text {dehydration }}\left(\mathrm{min}^{-1}\right)$ \\
\hline $0.13 \pm 0.005$ & $0.095 \pm 0.005$ & $0.002 \pm 0.0002$ \\
\hline & $\mathrm{T}=60^{\circ} \mathrm{C}, \mathrm{k}_{\text {maltose }}=\mathrm{k}_{1}$ \\
\hline
\end{tabular}

The $\mathrm{LiBr}$ AMSH solution is strongly acidic $(\mathrm{pH}<-2)$, which limits direct measurement of acid concentrations. Therefore, apparent rate constants are reported as described in the experimental section.

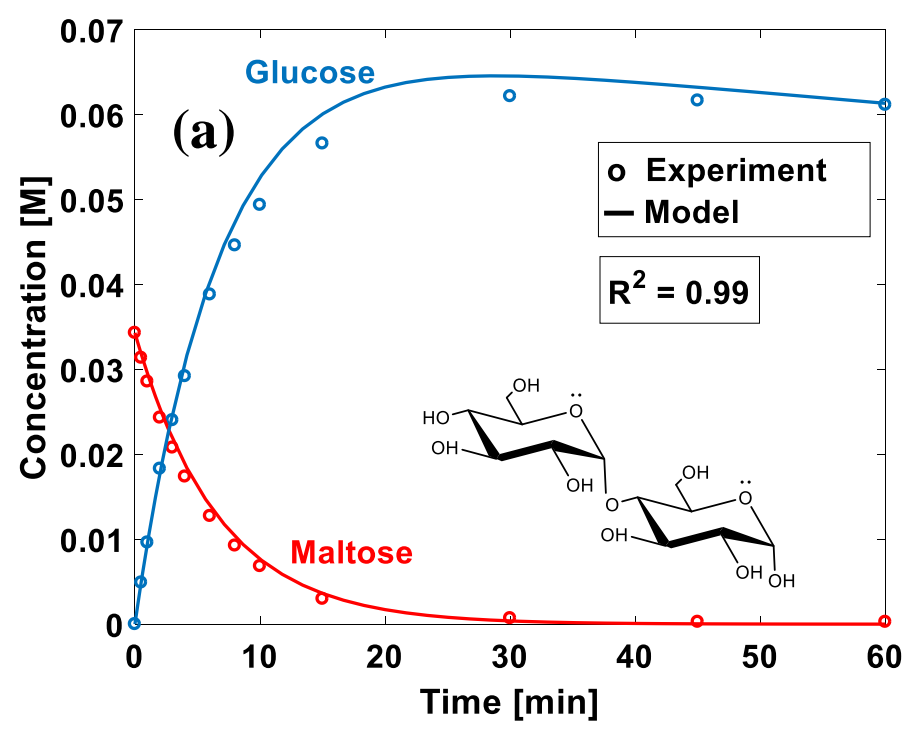




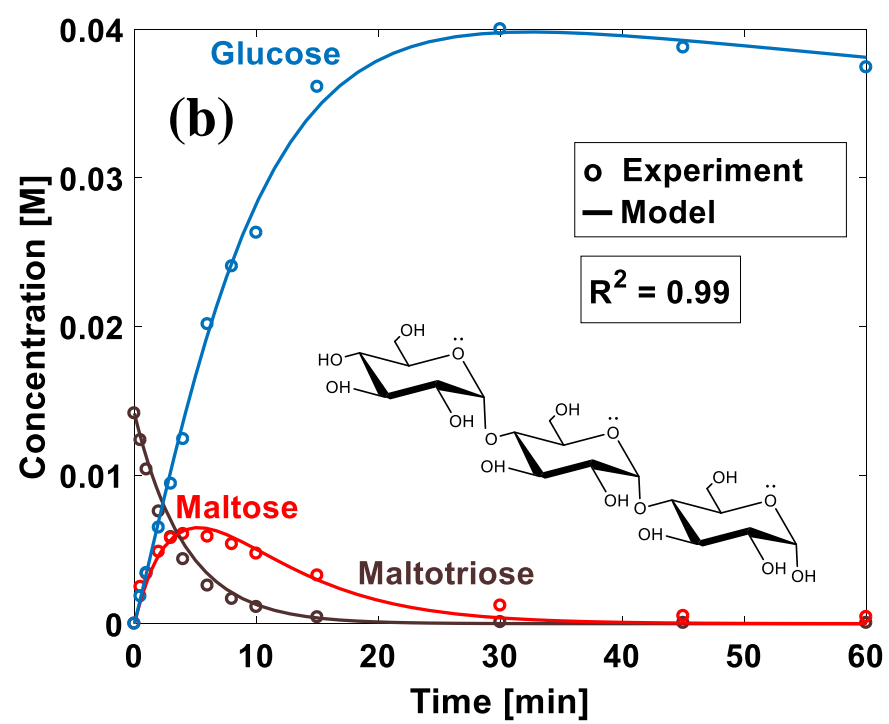

Figure 3.2: Time profiles of products from (a) maltose hydrolysis and (b) maltotriose hydrolysis in $\mathrm{LiBr}$ AMSH. Reaction conditions: $0.05 \mathrm{~g}$ of maltose or $0.033 \mathrm{~g}$ of maltotriose in $3.64 \mathrm{ml}$ of $\mathrm{LiBr}$ AMSH, $60{ }^{\circ} \mathrm{C}$. The model is regressed to these experimental data.

The rate constant $\mathrm{k}_{1}$ for bond scission at the non-reducing end is $\sim 1.4$ times larger than that of other glycosidic bonds $\mathrm{k}_{2}$. This ratio is in good agreement with the literature values (Table 1.1) for saccharide hydrolysis ${ }^{2,25-29,31}$. The rate constants $\mathrm{k}_{1}$ and $\mathrm{k}_{2}$ at different temperatures are provided in Table A.2. While both bonds possess similar apparent activation energies $\left(\sim 111 \pm 7 \mathrm{KJ} \mathrm{mol}^{-1}\right)$, the bond at the non-reducing end has a higher pre-exponential factor $\left(4.3 \times 10^{16} \mathrm{~min}^{-1}\right)$ compared to the other bonds $(3.2 \times$ $\left.10^{16} \mathrm{~min}^{-1}\right)$. This corroborates experimental results showing that the terminal bond at the reducing end hydrolyzes slower than the terminal bond at the non-reducing end ${ }^{26-31}$. Weintraub et al. ${ }^{26}$ observed that the lower entropy of activation of the reducing end bond reduces its tendency to undergo a transition to the half-chair conformation required to form the oxonium cation intermediate, which possesses high energy due to positive 
charge sharing and stabilization between the $\mathrm{C} 1$ carbon and the ring oxygen, in agreement with differences in our reported pre-exponential values. Hollo et al. ${ }^{34}$ found an increased reactivity of the non-reducing end due to its ability to convert to a more labile furanoside ring. The tendency of the reducing-glucan unit to convert to its open chain conformer allows for resonance between the cyclic and open form of the glucan residue and stabilizes the adjacent glycosidic bond. The glucose concentration reaches a maximum and then begins to decline slowly due to its dehydration to HMF (Figure 3.2). The difference in the maximum glucose yield for maltose and maltotriose is due to different starting amount of oligosaccharides. The glucose dehydration rate constant is very low $\left(0.002 \mathrm{~min}^{-1}\right)$ because of the low reaction temperature $\left(60{ }^{\circ} \mathrm{C}\right)$. Humins were not observed at this low reaction temperature.

Hydrolysis of maltotetraose (possessing two terminal and one interior bond) was conducted under similar reaction conditions as maltose and maltotriose to assess the predictive ability of the model. Rate constants $\left(\mathrm{k}_{1}\right.$ and $\left.\mathrm{k}_{2}\right)$ obtained from maltose and maltotriose hydrolysis kinetics were used to predict maltotetraose hydrolysis. Figure 3.3 shows reaction profiles for maltotetraose consumption and formation of products of DP from 1 to 4 . Very good agreement between the experimental and model predicted concentrations for all products is seen, as also indicated from the parity plot (Figure A.1). This result suggests that hydrolysis of any linear chain saccharide substrate can be described using the two fundamental bond scission rates. We provide further evidence of this finding below by studying starch. 


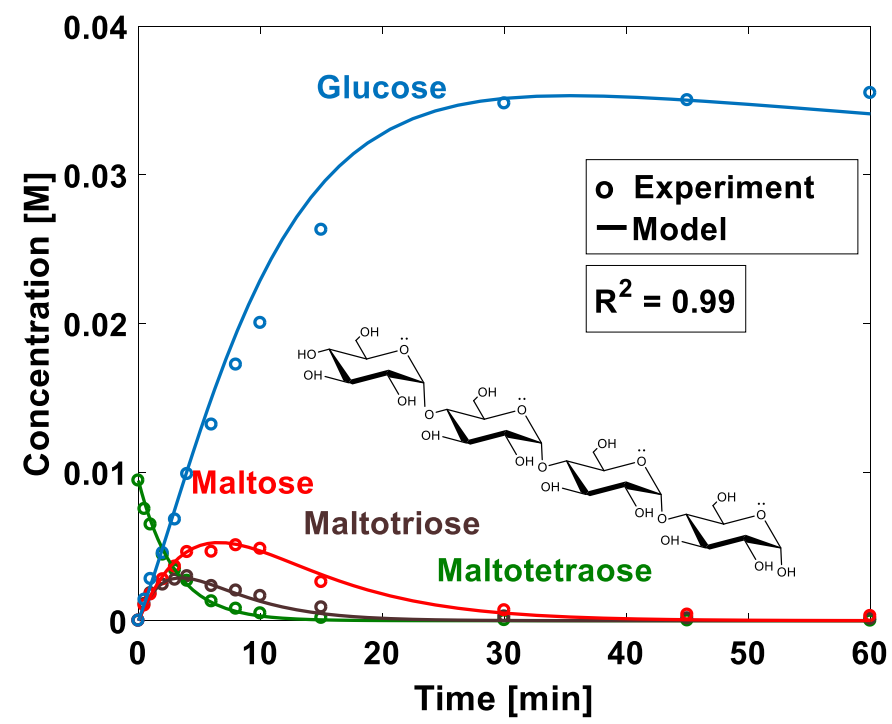

Figure 3.3: Time profile of maltotetraose hydrolysis in $\mathrm{LiBr}$ AMSH. Reaction conditions: $0.025 \mathrm{~g}$ of maltotetraose in $3.64 \mathrm{ml}$ of $\mathrm{LiBr} \mathrm{AMSH}, \mathrm{T}=60^{\circ} \mathrm{C}$.

\subsection{Hydrolysis of $\alpha$ - cyclodextrin}

The cyclodextrin consumption rate was obtained by monitoring its disappearance. Intermediates of DP of 1-5 were identified, quantified experimentally and compared with model predicted concentrations. The initial cyclodextrin consumption rate constant estimated $\left(0.14 \mathrm{~min}^{-1}\right)$ was lower than that expected for an oligosaccharide with 5 bonds $\left(0.13+(4 \times 0.095)=0.51 \mathrm{~min}^{-1}\right)$. The low consumption rate of the cyclodextrin compared to a linear saccharide could be as a result of an initial intra-molecular hydrogen bonding, which restricts hydrolysis of the cyclic saccharide. This additional resistance to hydrolysis due to hydrogen bonding in the cyclodextrin ring had been identified by Weintraub et al. ${ }^{26}$ They had estimated the activation energy for the acid hydrolysis of $\alpha(1-4)$ glycosidic bond in cyclodextrin ring to be 34.2 $\mathrm{kcal} / \mathrm{mole}$, while that for the hydrolysis of a linear oligosaccharide to be $30.5 \mathrm{kcal} / \mathrm{mole}$. This higher barrier for hydrolysis of cyclic oligosaccharide could be attributed to the 
energy required to overcome hydrogen bonding stabilizing the cyclic ring. They also found that the activation entropy of acid hydrolysis was $16.6 \mathrm{cal} /{ }^{\circ} \mathrm{C}$.mole for the linkages in the cyclic oligosaccharide, and $7.3 \mathrm{cal} /{ }^{\circ} \mathrm{C}$.mole for the linear oligosaccharides and attributed this to a transition from a very rigid cyclic structure to a more labile linear, open chain structure. These findings are consistent with our experimental observation of a slower hydrolysis rate in cyclodextrins. We hypothesize that, unlike linear oligosaccharides, cyclodextrin hydrolysis requires an initial ring opening step (which is the rate determining step) to form a linear chain, which then undergoes $\mathrm{C}-\mathrm{O}$ bond scission. Based on this hypothesis, we simulated $\alpha$-cyclodextrin hydrolysis using the model in Equations 3-1 \& 3-2.

$$
\begin{aligned}
& \text { Cyclodextrin } \stackrel{k_{\text {ring opening }}}{\longrightarrow} \text { Linear hexasaccharide } \\
& \text { Linear hexasaccharide } \stackrel{k_{1}+4 k_{2}}{\longrightarrow} \text { Glucose }
\end{aligned}
$$

The rate constant for ring opening was estimated to be $0.14 \mathrm{~min}^{-1}$. The model describes the experimental data reasonably for all intermediates and shows the initial delay in glucose formation (Figure 3.4, Figure A.2 and A.3). Maltohexaose could not be observed in the hydrolysis solution, as it coeluted with $\mathrm{LiBr}$ in the HPLC column. This initial delay in glucose formation is absent in the hydrolysis of linear saccharides, indicative of a ring opening step occuring in cyclodextrin before subsequent hydrolysis into glucose. 


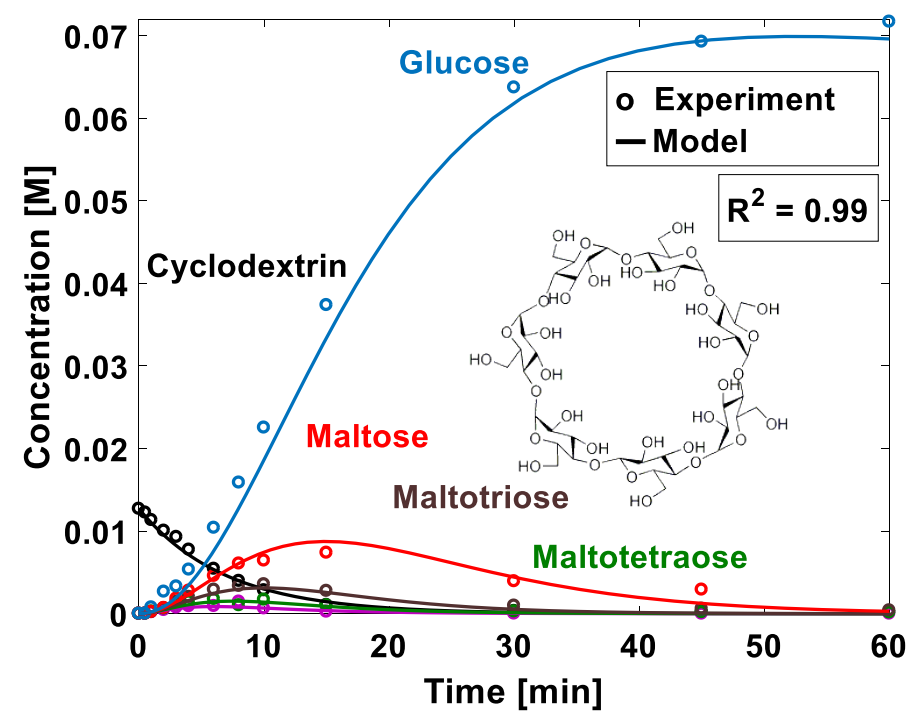

Figure 3.4: Time profile of $\alpha$ - cyclodextrin hydrolysis in LiBr AMSH. Reaction conditions: $0.05 \mathrm{~g}$ of $\alpha$ - cyclodextrin in $3.64 \mathrm{ml}$ of $\mathrm{LiBr}$ AMSH, $\mathrm{T}=60^{\circ} \mathrm{C}$. The ring opening rate constant is estimated with the other rate constants fixed.

\subsection{Hydrolysis of starch}

Next, we focus on the hydrolysis of potato starch that is composed of two main fractions: amylose and amylopectin. The amylose component is a linear chain polymeric material linked by $\alpha(1-4)$ glycosidic bonds, whereas amylopectin has a heavily branched polymeric structure composed of $\alpha(1-4)$ glycosidic bonds and an additional side of $\alpha(1-6)$ glycosidic bonds. The presence of highly structured amylopectin forms crystalline regions within the starch molecular structure. Figure 3.5 shows crystalline XRD peaks at $2 \theta$ of $5.35^{\circ}, 14.8^{\circ}, 17^{\circ}$, and $24^{\circ}$ which are characteristic of B - type starch granules ${ }^{44}$. Starch from tuber crops (yam, potato, sweet potato) have similar B-type classification ${ }^{45-47}$ due to the branching framework within amylopectin. The degree of 
crystallinity of potato starch is estimated as $29 \%$ based on the Scherrer equation, which is consistent with values reported in prior studies $45,46,48$.

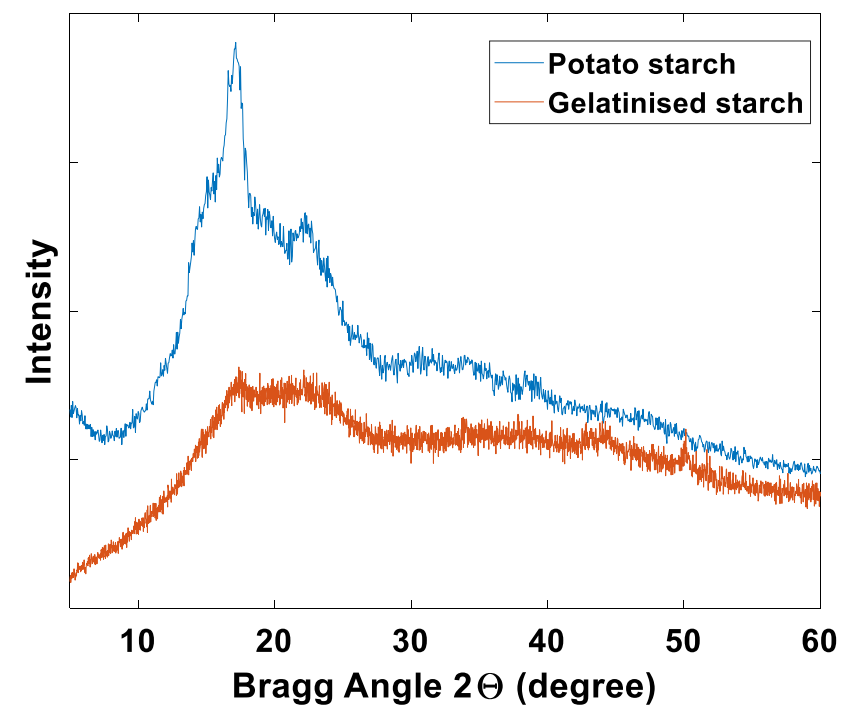

Figure 3.5: X-ray diffraction pattern of potato and gelatinised starch.

Conversion of crystalline regions to amorphous regions in potato starch (gelatinization) has been reported ${ }^{45,49}$ to occur around $65^{\circ} \mathrm{C}$. XRD after gelatinization shows that the crystalline peaks are completely absent (Figure 3.5). This suggests that starch gelatinization results in an amorphous material which can subsequently be easily hydrolyzed to glucose.

At temperatures below the gelatinization temperature, e.g., $60{ }^{\circ} \mathrm{C}$, the crystalline regions remain intact during reaction in the AMSH solution. This could explain the lower than expected conversion of potato starch to glucose at our reaction temperature (Figure 3.6), likely because of insufficient energy to disrupt the recalcitrant crystal framework of the crystalline regions. After $1 \mathrm{hr}$ of reaction, the temperature is set to 75 ${ }^{\circ} \mathrm{C}$. Temperature equilibration takes about $20 \mathrm{~min}$ and during this period, gelatinization and reaction probably happen. Upon reaching $75{ }^{\circ} \mathrm{C}$, starch hydrolysis resumes and the glucose yield increases (Figure 3.6). We hypothesize that this further glucose yield at 
$75^{\circ} \mathrm{C}$ comes from the unreacted crystalline starch which eventually becomes amorphous and hydrolyzes to glucose.

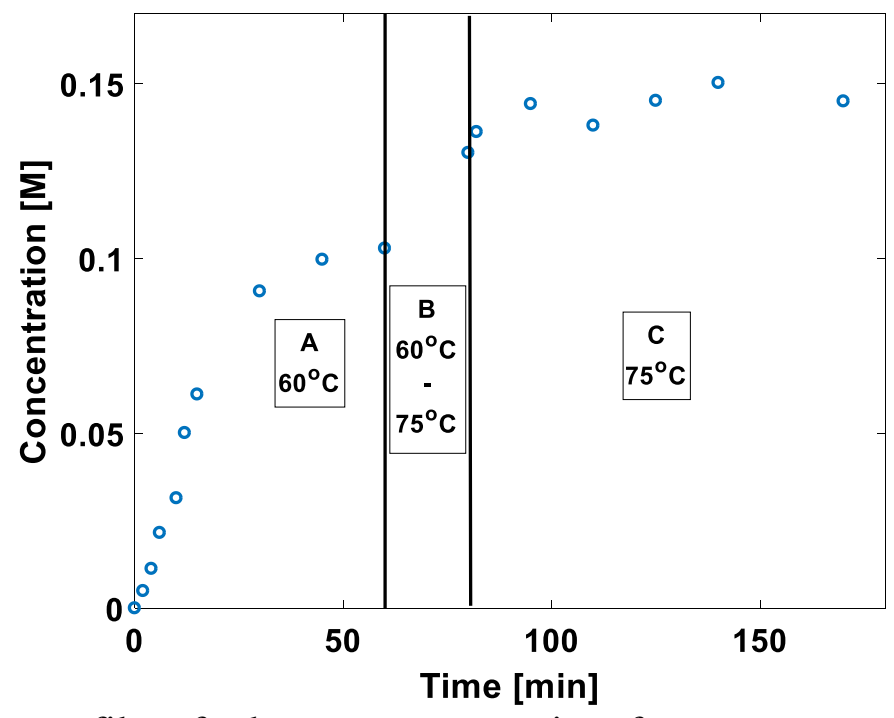

Figure 3.6: Time profile of glucose concentration from potato starch hydrolysis (amorphous + crystalline) in LiBr AMSH. Reaction conditions: $0.1 \mathrm{~g}$ of starch in 3.64 $\mathrm{ml}$ of $\mathrm{LiBr}$ AMSH, $\mathrm{T}=60^{\circ} \mathrm{C}$. A: amorphous region hydrolysis $\left(60^{\circ} \mathrm{C}\right)$. B: temperature equilibration from 60 to $75^{\circ} \mathrm{C} \mathrm{C}$ : crystalline region hydrolysis $\left(75^{\circ} \mathrm{C}\right)$.

To directly probe the presence of unconverted "crystalline" particulates in potato starch hydrolysis, we further used light scattering techniques. With static light scattering (Figure A.4), we measure the initial particle size distribution of potato starch in $\mathrm{LiBr}$ AMSH to be 45 microns, which is consistent with values reported in prior findings ${ }^{50}$. After hydrolysis of potato starch in LiBr AMSH for one hour, we identify particulates of 2 microns average size using DLS (Figure A.5). These particulates, which remain in AMSH solution at $60{ }^{\circ} \mathrm{C}$ (suspected to be crystalline starch remnants), convert to glucose above the gelatinization temperature (region $\mathrm{C}$ in Figure 3.6). 
For the simulation of amorphous starch hydrolysis, a linear polysaccharide of DP of 3000 was assumed. This DP was obtained by averaging values of DP reported in the literature for potato starch amylose $\mathrm{e}^{46,49,51}$. The amorphous content of starch, used in the simulation, was obtained by multiplying the mass of starch in the solution by the amorphous fraction index ( 0.7), obtained from XRD measurements. The model, with one fast scission linkage (rate constant $\mathrm{k}_{1}$ ) and the remaining slow scission linkages (rate constant $\mathrm{k}_{2}$ ), was used to simulate starch hydrolysis. Figure 3.7 compares experimentally quantified glucose

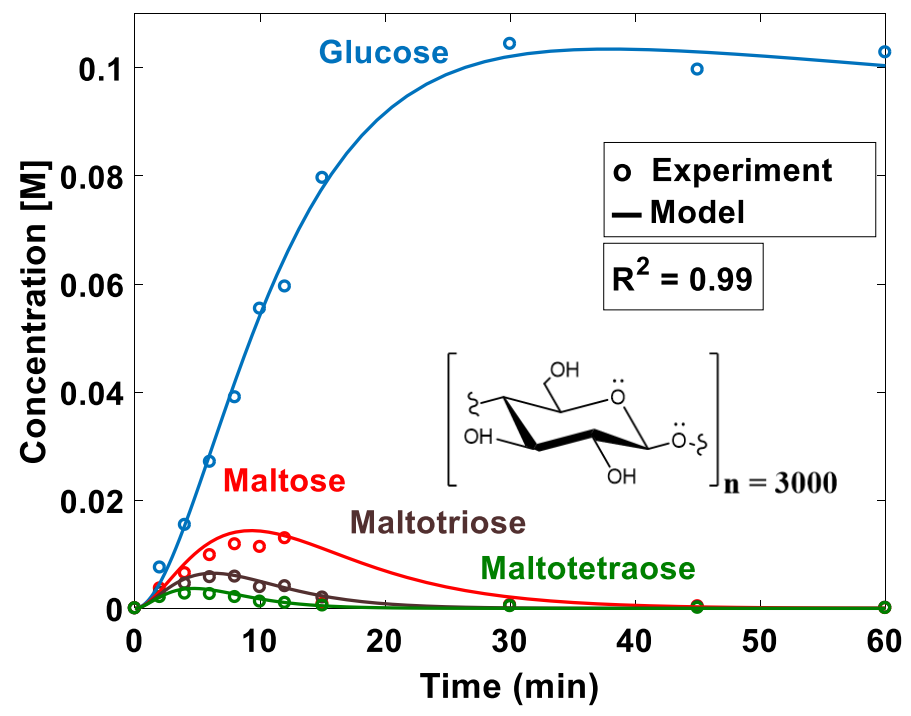

Figure 3.7: Time profile of products from potato starch hydrolysis in LiBr AMSH. Reaction conditions: $0.1 \mathrm{~g}$ of starch in $3.64 \mathrm{ml}$ of $\mathrm{LiBr} \mathrm{AMSH}, \mathrm{T}=60{ }^{\circ} \mathrm{C}$. Lines are model predictions and points are experimental data.

and oligosaccharides concentrations with the model predicted values. While oligosaccharides of DP only up to 4 were quantified experimentally, due to available standards for calibration, the model described all saccharides up to $\mathrm{n}=3000$. Experimental quantification of oligosaccharides of DP $\geq 6$ by the chromatographic 
separation method is challenging. The model circumvents this challenge and predicts time dependent concentrations (Figure 3.7) for saccharides produced during the reaction. The parity plot in Figure A.6 suggests good agreement between model and experiments

\subsection{Effect of saccharide chain length on hydrolysis}

Taken all together, a model that includes scission of the non-reducing terminal (fast scission, $\mathrm{k}_{1}$ ) and of all other linkages (slow scission, $\mathrm{k}_{2}$ ) and potentially a ring opening step, if needed, can describe the hydrolysis of saccharides of any chain length and chain configuration (cyclic or open chain). Next, the effect of chain length on saccharide hydrolysis rate constant and the yield of glucose were elucidated. As seen in Figure 3.8a, Figure A.7 and Equation 3-3, the substrate consumption rate constant, which has been described as first order with respect to the number of glycosidic bonds (n-1) in a saccharide $2,27,52,53$, increases with increasing chain length

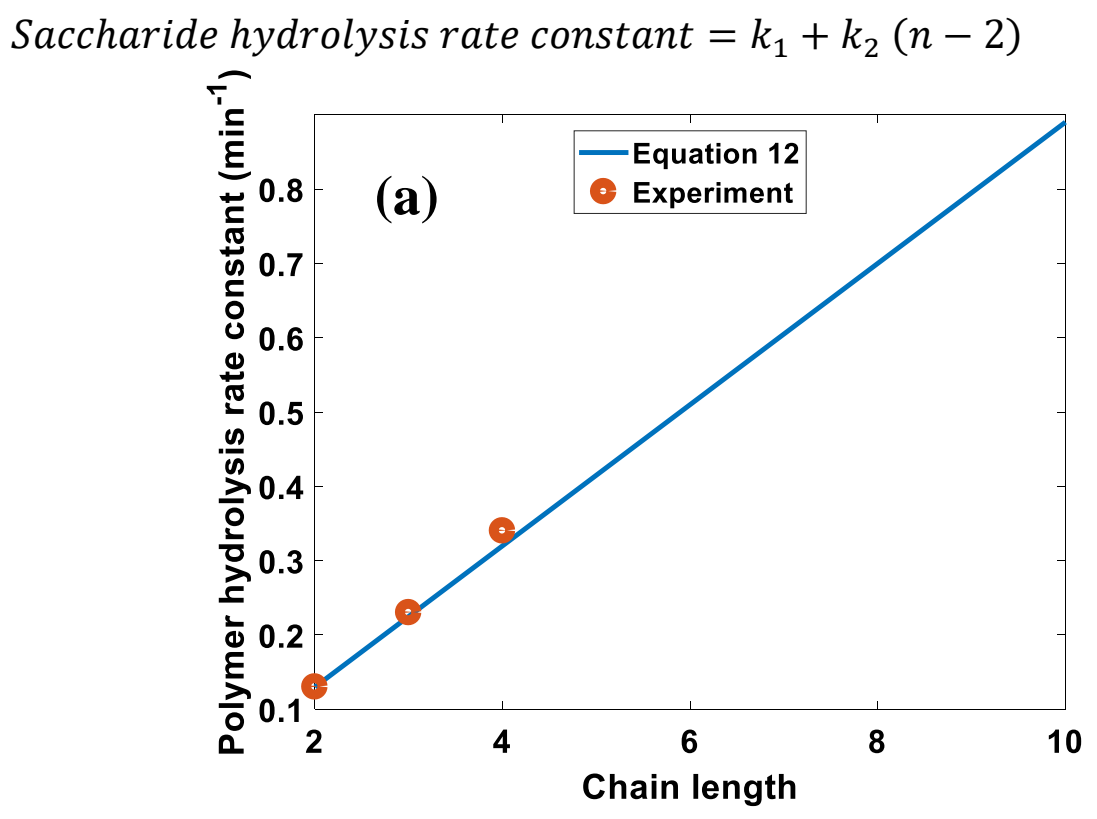




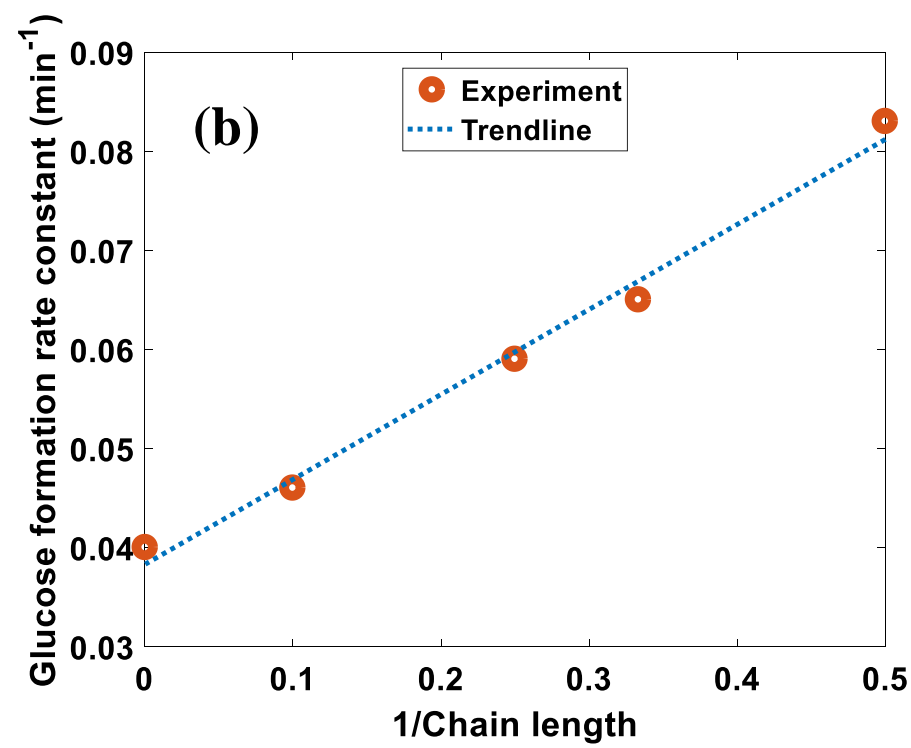

Figure 3.8: Effect of chain length on (a) saccharide hydrolysis rate constant and (b) glucose formation rate constant (trendline shows inverse relation). Points are experimentally determined rate constants. Reaction conditions: $0.1 \mathrm{~g}$ of oligosaccharide in $3.64 \mathrm{ml} \mathrm{LiBr} \mathrm{AMSH}, \mathrm{T}=60^{\circ} \mathrm{C}$.

Interestingly, an inverse trend is observed when the glucose formation rate constant (see Equations $3 \& 4$ ) is plotted vs. chain length (Figure 3.8b and Figure A.8). This decrease in glucose formation rate constant with chain length can be explained by comparing the time taken to hydrolyze bonds in different saccharides. For example, comparing bond scission in maltose to maltotriose, each bond scission in maltose directly forms two molecules of glucose with an average characteristic time of $\frac{1}{k_{1}}$. For maltotriose hydrolysis to glucose, two different paths exist depending on which of the two bonds is protonated (and subsequently hydrolyzed). The average characteristic time for these two paths is longer than that for maltose. This is due to the fact that for long chains, protonation does not happen at all bond sites at an instance $25,42,43$ and thus, only the protonated bonds can by hydrolyzed. In contrast, for disaccharides, all molecules 
can be protonated at the same time (at least in the continuum limit) and glucose forms faster. This characteristic time for complete saccharide scission to glucose keeps increasing with chain length, as more intermediate species form on their way to glucose. For the reasons discussed above, the fraction to glucose produced from saccharide hydrolysis decreases as a function of chain length. It also implies that glycosidic bonds are cleaved randomly, rather than in a stepwise manner from one chain end to the other.

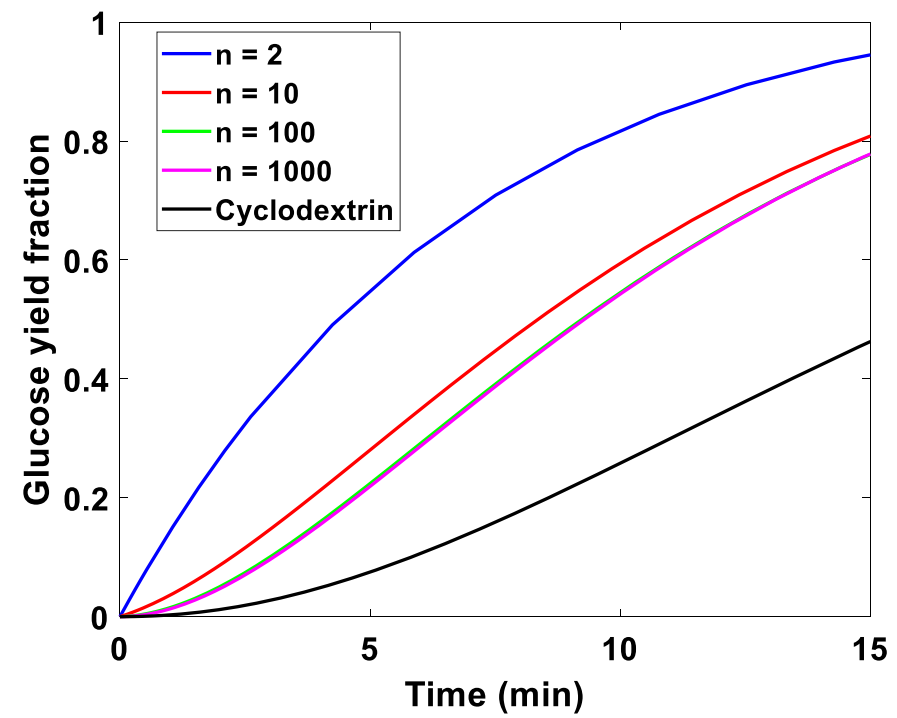

Figure 3.9: Simulated glucose formation profile from hydrolysis of oligosaccharides or polysaccharides of different chain length. Reaction conditions: $0.1 \mathrm{~g}$ of oligosaccharide in $3.64 \mathrm{ml}$ of $\mathrm{LiBr} \mathrm{AMSH}, \mathrm{T}=60{ }^{\circ} \mathrm{C}$.

Figure 3.9 shows an initial delay time in glucose formation from oligosaccharides or polysaccharides of $n \geq 10$. A similar trend was observed for cyclodextrin hydrolysis. This delay arises from multiple bond scission steps occurring before forming glucose: polysaccharide $\rightarrow$ short chain polysaccharide $\rightarrow$ oligosaccharide $\rightarrow$ glucose. The initial delay becomes more evident with an increase in the chain length of linear saccharides, which results in lower glucose formation in the 
early stages of hydrolysis. These results are in agreement with findings ${ }^{42}$ by Vanoye et al. where the initial delay in glucose formation from cellulose was attributed to random scission along the saccharide chain.

Importantly, the conversion of all substrates achieved $>90 \%$ glucose yields within 30 minutes. This is due to a strong acidic environment created by LiBr AMSH which also prevents the retrogradation of long chain saccharides. Long chain saccharide retrogradation had been identified by Pinto et $a l^{25}$. Retrogradation occurs due to the alignment of linear chains resulting in hydrogen bond formation and subsequently weak crystalline centers between chains. LiBr AMSH, which has been shown to be effective in distorting hydrogen bond interactions ${ }^{5,38}$ in cellulose and in facilitating its hydrolysis to glucose, prevents retrogradation of long linear saccharide chains. Because saccharide hydrolysis follows similar mechanism in pure acid ${ }^{24,28}$, ionic liquids ${ }^{2}$ and acidified molten salt hydrate, our mass action kinetic model could be applicable to a broad saccharide hydrolysis chemistry in Brønsted acid media. 


\section{Chapter 4}

\section{CONCLUSIONS}

This work focused on the kinetics of starch-saccharified glucose production from food waste as a possible precursor for renewable chemicals and fuels. We studied experimentally hydrolysis kinetics of $\alpha(1-4)$ glycosidic bonds of small linear and cyclic oligosaccharides and actual potato starch in $\mathrm{LiBr}$ AMSH and developed the first kinetic model that follows mass action kinetics. The model parameters are estimated from the kinetics data of maltose, maltotriose and cyclodextrin and then used to predict the rest. The experimentally measured concentrations agree well with the model predictions. We find that all acyclic saccharides can be described with two rate constants only. The non-reducing terminal bonds hydrolyze faster than all other bonds including the reducing terminal bonds within a saccharide chain. The hydrolysis of cyclodextrin progresses via an initial ring-opening followed by scission of all internal bonds. The hydrolysis rate constant of linear saccharides is directly proportional to the substrate chain length, whereas the glucose formation rate constant is inversely proportional to the chain length. Hydrolysis of potato starch, a complex carbohydrate containing both crystalline and amorphous starch, progresses by deconstruction of the amorphous phase at $60{ }^{\circ} \mathrm{C}$. The crystalline starch gelatinizes to amorphous at higher temperatures, e.g., $65^{\circ} \mathrm{C}$, and thereafter it hydrolyzes to glucose. The AMSH is a very effective medium for short processing times and high glucose yields. A two-step modeling approach - heterogeneous shrinking particle step and hydrolysis - will be

necessary for the kinetic model of cellulose with $\beta$-glycosidic bonds because of its dissolution limitation in aqueous solvent. 


\section{REFERENCES}

1. Climent, M. J., Corma, A. \& Iborra, S. Converting carbohydrates to bulk chemicals and fine chemicals over heterogeneous catalysts. Green Chem. 13, 520-540 (2011).

2. Enslow, K. R. \& Bell, A. T. The kinetics of Brønsted acid-catalyzed hydrolysis of hemicellulose dissolved in 1-ethyl-3-methylimidazolium chloride $\{$. RSC Adv. 2, 10028-10036 (2012).

3. Pacific Northwest National Laboratory (PNNL \& National Renewable Energy Laboratory (NREL). Top Value Added Chemicals from Biomass Volume IResults of Screening for Potential Candidates from Sugars and Synthesis Gas Energy Efficiency and Renewable Energy. (2004).

4. Sadula, S., Oesterling, O., Nardone, A., Dinkelacker, B. \& Saha, B. One-pot integrated processing of biopolymers to furfurals in molten salt hydrate: understanding synergy in acidity. Green Chem. 19, 3888-3898 (2017).

5. Sadula, S., Athaley, A., Zheng, W., Ierapetritou, M. \& Saha, B. Process Intensification for Cellulosic Biorefineries. ChemSusChem 10, 2566-2572 (2017).

6. Chheda, J. N., Roman-Leshkov, Y. \& Dumesic, J. a. Production of 5hydroxymethylfurfural and furfural by dehydration of biomass-derived monoand poly-saccharides. Green Chem. 9, 342 (2007).

7. Lee, Y. Y., Prashant, I. \& Torget, R. W. Dilute-Acid Hydrolysis of Lignocellulosic Biomass. Adv. Biochem. Eng. Biotechnol. 65, 94-115 (1999).

8. Yoo, C. G., Zhang, S. \& Pan, X. Effective conversion of biomass into bromomethylfurfural, furfural, and depolymerized lignin in lithium bromide molten salt hydrate of a biphasic system. RSC $A d v$. 7, 300-308 (2017).

9. Mante, Ofei D; Agblevor, F. A. Catalytic conversion of biomass to biofuels. Biomass Conv. Bioref. 1, 203-215 (2011).

10. Tang, X. et al. Green Processing of Lignocellulosic Biomass and Its Derivatives in Deep Eutectic Solvents. ChemSusChem 1-12 (2017). doi: $10.1002 /$ cssc. 201700457

11. Yang, L. et al. One-pot synthesis of 5-hydroxymethylfurfural from carbohydrates using an inexpensive $\mathrm{FePO}_{4}$ catalyst. RSC Adv. 5, 19900-19906 (2015).

12. $\mathrm{Yu}, \mathrm{I}$. K. M. et al. Catalytic valorization of starch-rich food waste into hydroxymethylfurfural (HMF): Controlling relative kinetics for high productivity. Bioresour. Technol. 237, 222-230 (2017).

13. Saeman, J. F. Kinetics of Wood Saccharification - Hydrolysis of Cellulose and Decomposition of Sugars in Dilute Acid at High Temperature. Ind. Eng. Chem. 37, 43-52 (1945).

14. Fagan, R. D., Grethlein, H. E., Converse, A. O. \& Porteous, A. Kinetics of the Acid Hydrolysis of Cellulose Found in Paper Refuse. JACS 5, 545-547 (1971).

15. Girisuta, B., Janssen, L. P. B. M. \& Heeres, H. J. Kinetic Study on the Acid- 
Catalyzed Hydrolysis of Cellulose to Levulinic Acid. Ind. Eng. Chem. Res. 46, 1696-1708 (2007).

16. Xiang, Q., Seok Kim, J. \& Lee, Y. Y. Model for Dilute-Acid Hydrolysis of Cellulose A Comprehensive Kinetic Model for Dilute-Acid Hydrolysis of Cellulose. Appl. Biochem. Biotechnol. 105-108, 337-352 (2003).

17. Dadach, Z.-E. \& Kaliaguine, S. Acid hydrolysis of cellulose. Part 1. Experimental kinetic analysis. Can. J. Chem. Eng. 71, 880-891 (1993).

18. Kupiainen, L., Ahola, J. \& Tanskanen, J. Kinetics of Formic Acid-catalyzed Cellulose Hydrolysis. BioResources 9, 2645-2658 (2014).

19. Huang, Y.-B. \& Fu, Y. Hydrolysis of cellulose to glucose by solid acid catalysts. Green Chem. 15, 1095 (2013).

20. Li, J., Zhang, X., Zhang, M., Xiu, H. \& He, H. Optimization of Selective Acid Hydrolysis of Cellulose for Microcrystalline Cellulose using FeCl3.

BioResources 9, 1334-1345 (2014).

21. Lin, C. S. K. et al. Food waste as a valuable resource for the production of chemicals, materials and fuels. Current situation and global perspective. Energy Environ. Sci. 6, 426-464 (2013).

22. Mirabella, N., Castellani, V. \& Sala, S. Current options for the valorization of food manufacturing waste: A review. J. Clean. Prod. 65, 28-41 (2014).

23. Lin, C. S. K. et al. Current and future trends in food waste valorization for the production of chemicals, materials and fuels: a global perspective. Biofuels, Bioprod. Biorefining 8, 686-715 (2014).

24. Bemiller, J. N. Acid -Catalyzed Hydrolysis of Glycosides. in Advances in Carbohydrate Chemistry (eds. Wolfrom, M. L. \& Tipson, R. S.) (Academic Press Inc., 1967).

25. Pinto, J. Q. \& Kaliaguine, S. A Monte Carlo analysis of acid hydrolysis of glycosidic bonds in polysaccharides. AIChE J. 37, 905-914 (1991).

26. Weintraub, M. S. \& French, D. Acid Hydrolysis of (1-4)-alpha-D-Glucans. Part II. Carbohydr. Res. 15, 251-262 (1970).

27. Beltrame, P. L. et al. Kinetics of enzymic hydrolysis of malto-oligosaccharides: A comparison with acid hydrolysis. Carbohydr. Res. 166, 71-83 (1987).

28. Kamiyama, Y. \& Sakai, Y. Rate of hydrolysis of xylo-oligosaccharides in dilute sulfuric acid. Carbohydr. Res. 73, 151-158 (1979).

29. Weintraub, M. S. \& French, D. Acid Hydrolysis of (1-4)-alpha-D-Glucans. Part I. Carbohydr. Res. 15, 241-250 (1970).

30. Weintraub, M. S. Physical chemical studies on amylose. Dissertation (Iowa State University, 1967).

31. Feather, M. S. \& Harris, J. F. Partial Hydrolysis and Acetolysis of Cellotriose1-C14. J. Am. Chem. Soc. 89, 5661-5664 (1967).

32. Freudenberg, K. \& Blomqvist, G. Die Hydrolyse der Cellulose und ihrer Oligosaccharide. Berichte der Dtsch. Chem. Gesellschaft 2070-2082 (1935).

33. Nguyen, H. S. H., Heinonen, J. \& Sainio, T. Acid hydrolysis of glycosidic bonds in oat B-glucan and development of a structured kinetic model. AIChE J. 
64, 2570-2580 (2018).

34. Hollo, J. \& Laszlo, E. Kinetical comparison of the acidic, alkaline and enzymatic hydrolysis of starch. Period. Polytech. Chem. Eng. 14, 33-46 (1970).

35. Leipner, H., Fischer, S., Brendler, E. \& Voigt, W. Structural changes of cellulose dissolved in molten salt hydrates. Macromol. Chem. Phys. 201, 20412049 (2000).

36. Van Den Bergh, J., Babich, I. V., O’Connor, P. \& Moulijn, J. A. Production of Monosugars from Lignocellulosic Biomass in Molten Salt Hydrates: Process Design and Techno-Economic Analysis. Ind. Eng. Chem. Res. 56, 13423-13433 (2017).

37. Li, J., Spina, A., Moulijn, J. A. \& Makkee, M. Sorbitol dehydration into isosorbide in a molten salt hydrate medium. Catal. Sci. Technol. 3, 1540 (2013).

38. Deng, W., Kennedy, J. R., Tsilomelekis, G., Zheng, W. \& Nikolakis, V. Cellulose Hydrolysis in Acidified LiBr Molten Salt Hydrate Media. Ind. Eng. Chem. Res. 54, 5226-5236 (2015).

39. Franzyshen, S. K., Schiavelli, M. D., Stocker, K. D. \& Ingram, M. D. Proton acidity and chemical reactivity in molten salt hydrates. J. Phys. Chem. 94, 2684-2688 (1990).

40. Li, J., Soares, H. S. M. P., Moulijn, J. a. \& Makkee, M. Simultaneous hydrolysis and hydrogenation of cellobiose to sorbitol in molten salt hydrate media. Catal. Sci. Technol. 3, 1565 (2013).

41. Yan, L., Greenwood, A. a., Hossain, A. \& Yang, B. A comprehensive mechanistic kinetic model for dilute acid hydrolysis of switchgrass cellulose to glucose, 5-HMF and levulinic acid. RSC Adv. 4, 23492 (2014).

42. Vanoye, L., Fanselow, M., Holbrey, J. D., Atkins, M. P. \& Seddon, K. R. Kinetic model for the hydrolysis of lignocellulosic biomass in the ionic liquid, 1-ethyl-3-methyl-imidazolium chloride. Green Chem. 11, 390-396 (2009).

43. Joksimovic, G. \& Markovic, Z. Investigation of the Mechanism of Acidic Hydrolysis of Cellulose. Acta Agric. Serbica 24, 51-57 (2007).

44. Hoover, R. \& Vasanthan, T. Effect of heat-moisture treatment on the structure and physicochemical properties of cereal, legume, and tuber starches. Carbohydr. Res. 252, 33-53 (1994).

45. Nakamura, Y. Starch: Metabolism and structure. Starch: Metabolism and Structure (Springer, 2015). doi:10.1007/978-4-431-55495-0

46. Zobel, H. Molecules to Granules: A Comprehensive Starch Review. Die Starke 40, 44-50 (1988).

47. Kainuma, K. \& French, D. Nageli amylodextrin and its relationship to starch granule structure. I. Preparation and properties of amylodextrins from various starch types. Biopolymers 10, 1673-1680 (1971).

48. Nara, S. \& Komiya, M. Study on Relative Crystallinity of Moist Potato Starch. Starch 30, 111-114 (1978).

49. Bertoft, E. \& Blennow, A. Structure of Potato Starch. Adv. Potato Chem. Technol. Second Ed. 57-73 (2016). doi:10.1016/B978-0-12-800002-1.00003-0 
50. Dhital, S., Shrestha, A. K. \& Gidley, M. J. Relationship between granule size and in vitro digestibility of maize and potato starches. Carbohydr. Polym. 82, 480-488 (2010).

51. Bertoft, E. Analysing starch structure. Starch Food Struct. Funct. Appl. 57-96 (2004). doi:10.1016/B978-1-85573-731-0.50006-2

52. Sharples, A. Degradation of Cellulose and Its Derivatives: A. Acid Hydrolysis and Alcoholysis. in Cellulose and Cellulose Derivatives (eds. Bikales, N. M. \& Segal, L.) (Wiley-Interscience, 1968).

53. Ladisch, M. R. Hydrolysis. in Biomass Handbook (eds. Kitani, O. \& Hall, C. W.) (Gordon and Breach, 1989).

54. Camacho, F., González-Tello, P., Jurado, E. \& Robles, A. Microcrystallinecellulose hydrolysis with concentrated sulphuric acid. J. Chem. Technol. Biotechnol. 67, 350-356 (1996).

55. Dee, S. J. \& Bell, A. T. A study of the acid-catalyzed hydrolysis of cellulose dissolved in ionic liquids and the factors influencing the dehydration of glucose and the formation of humins. ChemSusChem 4, 1166-1173 (2011). 


\section{Appendix}

\section{TABLES AND FIGURES}

Table A.1: Comparison of maximum glucose yields in different hydrolysis medium

\begin{tabular}{|c|c|c|c|c|c|}
\hline & Medium & Temperature & $\begin{array}{l}\text { Time } \\
\text { (mins) } \\
\end{array}$ & $\begin{array}{c}\text { Maximum } \\
\text { glucose yield } \\
\end{array}$ & Reference \\
\hline \multirow[t]{3}{*}{ Dilute Acid } & $0.05-1 \mathrm{M}$ & & & & \\
\hline & $\mathrm{H}_{2} \mathrm{SO}_{4}$ & $150-200{ }^{\circ} \mathrm{C}$ & 30 & 30 & 15 \\
\hline & $0.2 \mathrm{M} \mathrm{H}_{2} \mathrm{SO}_{4}$ & $140-220^{\circ} \mathrm{C}$ & 60 & 46 & 41 \\
\hline Concentrated & $13.5 \mathrm{M} \mathrm{H}_{2} \mathrm{SO}_{4}$ & $40{ }^{\circ} \mathrm{C}$ & 720 & 32 & 54 \\
\hline Acid & $12 \mathrm{M} \mathrm{H}_{2} \mathrm{SO}_{4}$ & $40{ }^{\circ} \mathrm{C}$ & 1200 & 18 & 54 \\
\hline Ionic & $\mathrm{C} 2 \operatorname{mim}[\mathrm{Cl}]+$ & & & & \\
\hline \multirow[t]{4}{*}{ Liquids } & $0.23 \mathrm{M}$ & & & & \\
\hline & $\mathrm{CH}_{3} \mathrm{SO}_{3} \mathrm{H}$ & $90^{\circ} \mathrm{C}$ & 120 & 50 & 42 \\
\hline & $\operatorname{Bmim}[\mathrm{Cl}]+0.03$ & & & & \\
\hline & $\mathrm{M} \mathrm{H}_{2} \mathrm{SO}_{4}$ & $90^{\circ} \mathrm{C}$ & 300 & 35 & 55 \\
\hline Acidified & LiBr AMSH & $85^{\circ} \mathrm{C}$ & 40 & 82 & 38 \\
\hline \multirow[t]{2}{*}{$\mathrm{MSH}$} & $\mathrm{ZnCl}_{2} \mathrm{MSH}+$ & & & & \\
\hline & $0.4 \mathrm{M} \mathrm{HCl}$ & $70{ }^{\circ} \mathrm{C}$ & 60 & 85 & 36 \\
\hline
\end{tabular}

Table A.2: Rate constants $\mathrm{k}_{1}$ and $\mathrm{k}_{2}$ at different temperatures

\begin{tabular}{rcc} 
& $\mathrm{k}_{1}\left(\mathrm{~min}^{-1}\right)$ & $\mathrm{k}_{2}\left(\mathrm{~min}^{-1}\right)$ \\
\hline $60{ }^{\circ} \mathrm{C}$ & 0.13 & 0.095 \\
$50{ }^{\circ} \mathrm{C}$ & 0.044 & 0.034 \\
$40{ }^{\circ} \mathrm{C}$ & 0.01 & 0.0079 \\
\hline
\end{tabular}



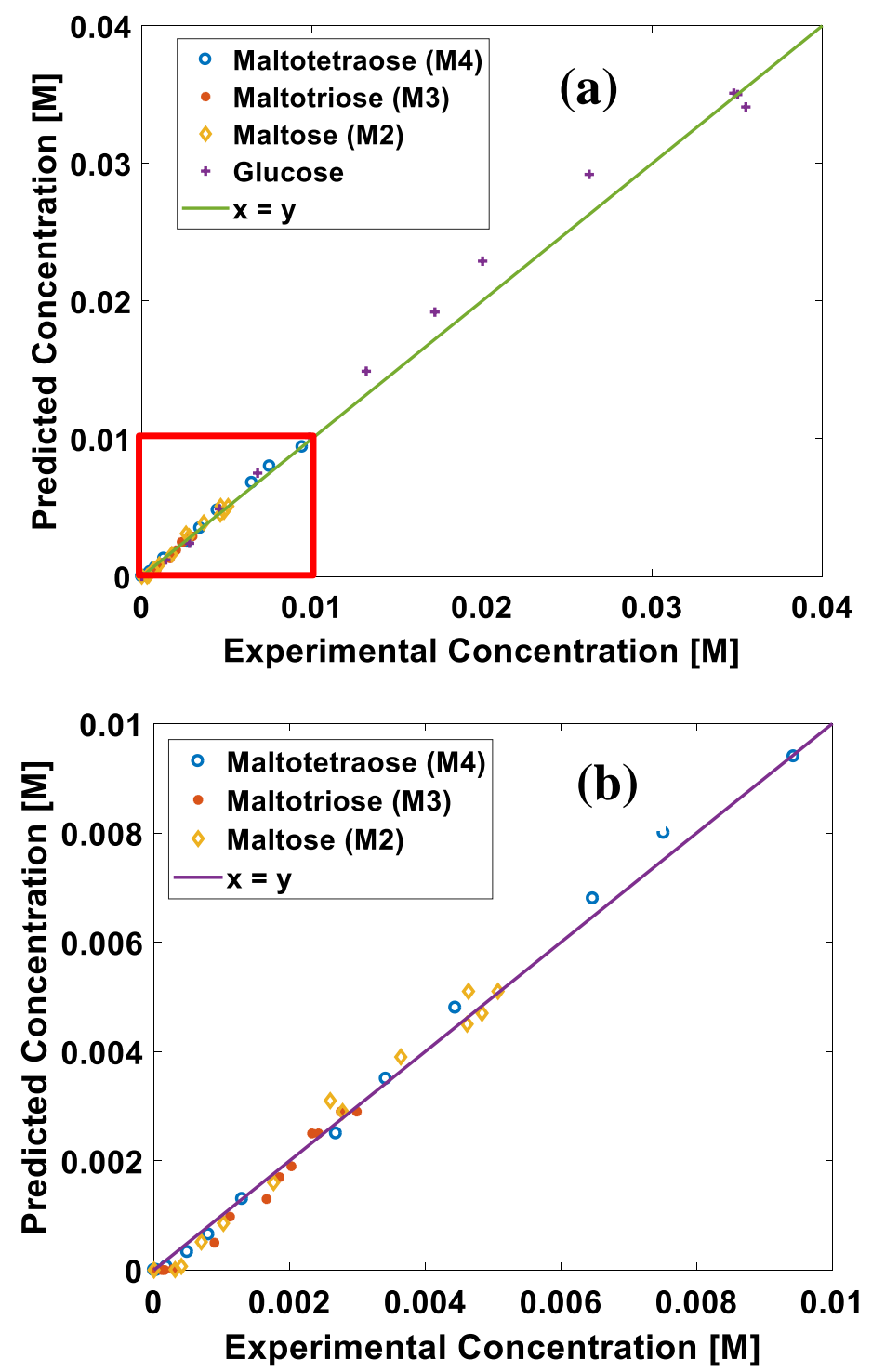

Figure A.1: Parity plot of predicted vs. experimental data from maltotetraose hydrolysis in LiBr AMSH. a) All reaction products. b) Zoomed inset. Reaction conditions: $0.025 \mathrm{~g}$ of maltotetraose in $3.64 \mathrm{ml}$ of $\mathrm{LiBr} \mathrm{AMSH}, \mathrm{T}=60^{\circ} \mathrm{C}$. 


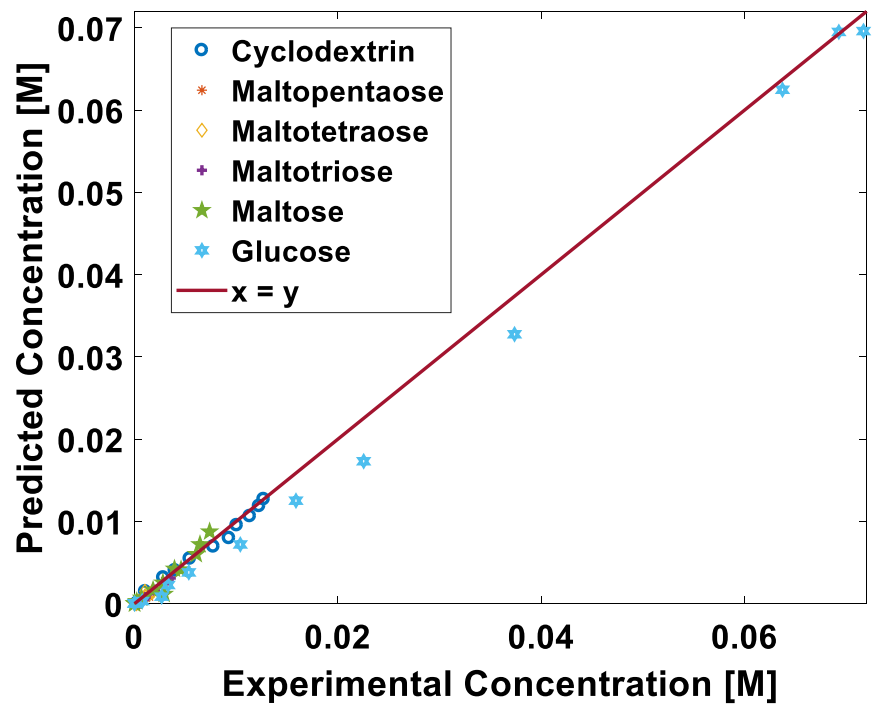

Figure A.2: Predicted vs. experimental data from $\alpha$-cyclodextrin hydrolysis in $\mathrm{LiBr}$ AMSH for all reaction products. Reaction conditions: $0.05 \mathrm{~g}$ of $\alpha$-cyclodextrin in 3.64 $\mathrm{ml}$ of $\mathrm{LiBr} \mathrm{AMSH}, \mathrm{T}=60{ }^{\circ} \mathrm{C}$.

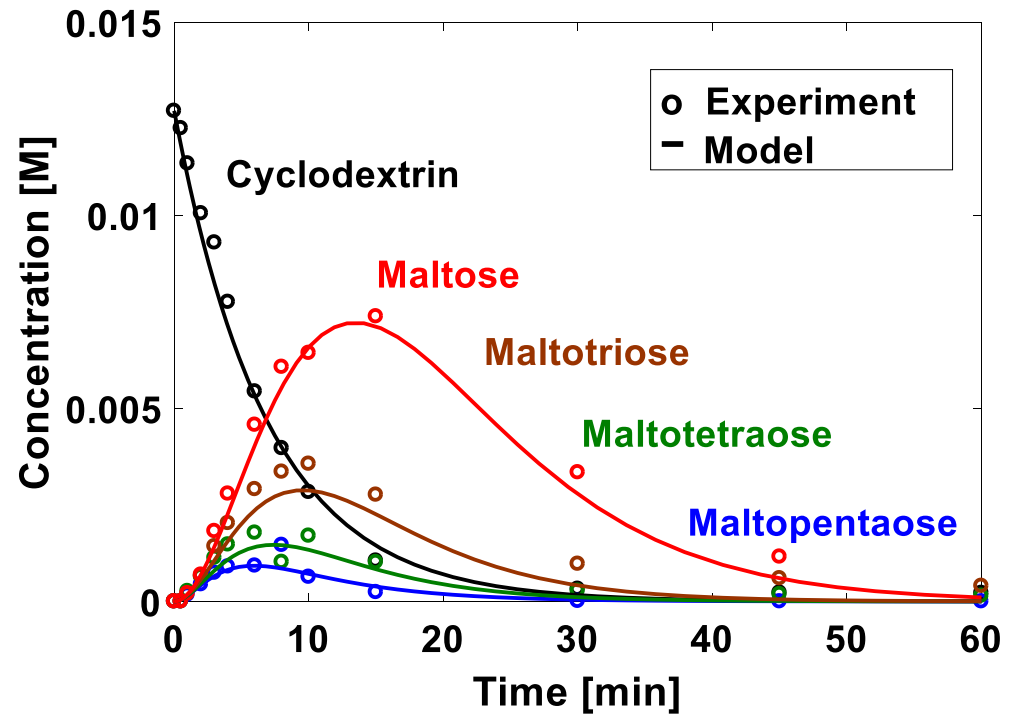

Figure A.3: Time dependent profile of observable intermediates from $\alpha$-cyclodextrin hydrolysis in $\mathrm{LiBr}$ AMSH. Reaction conditions: $0.05 \mathrm{~g}$ of $\alpha$-cyclodextrin in $3.64 \mathrm{ml}$ of LiBr AMSH, $\mathrm{T}=60{ }^{\circ} \mathrm{C}$. 


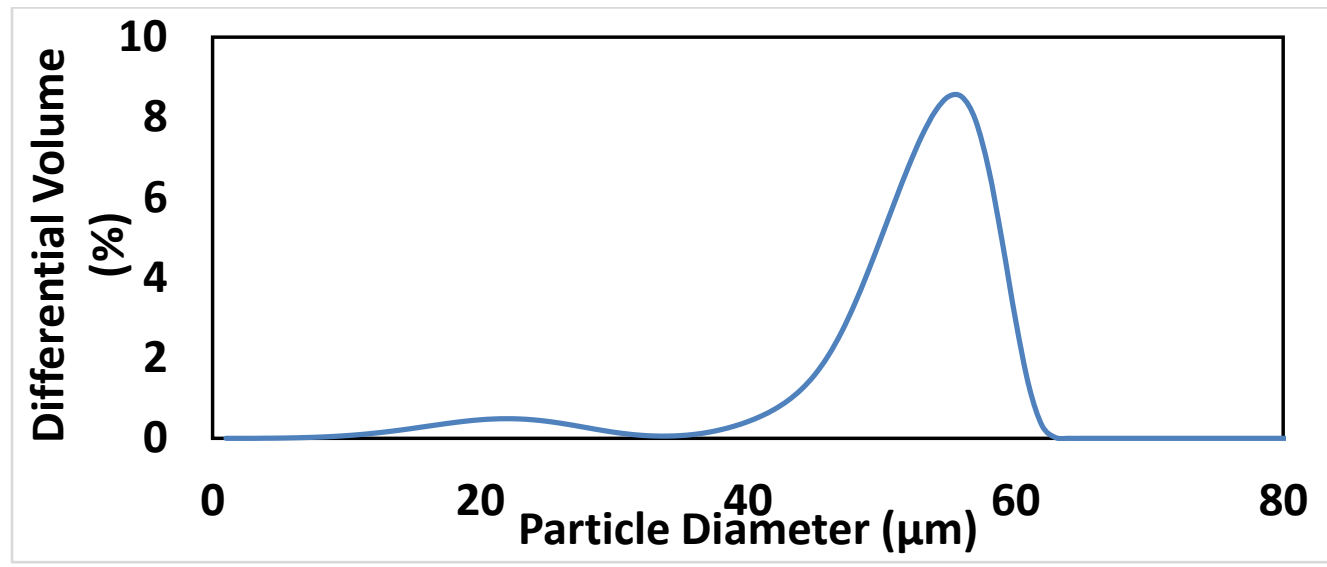

Figure A.4: Particle size distribution of potato starch in LiBr MSH (before hydrolysis) using SLS.

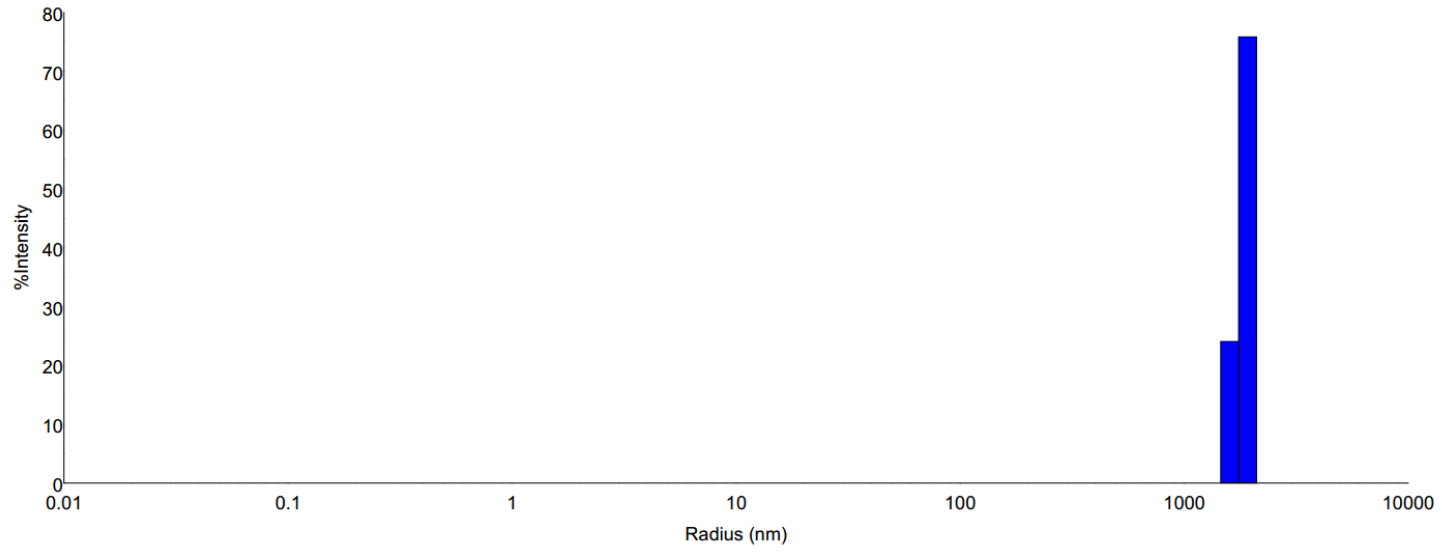

Figure A.5: Particle size distribution of potato starch in LiBr AMSH after hydrolysis using DLS. 


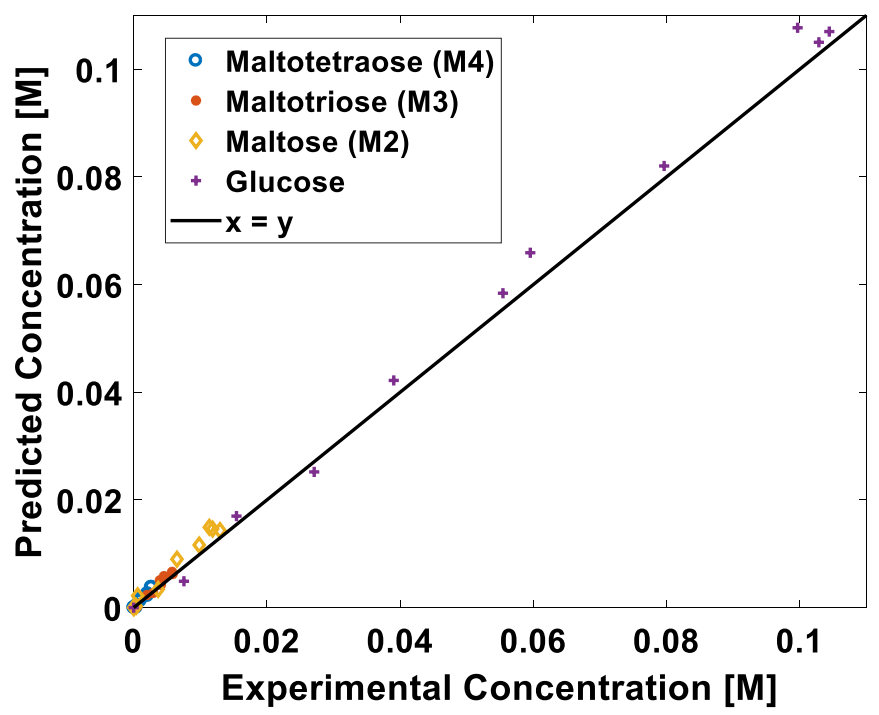

Figure A.6: Parity plot of predicted vs. experimental concentrations of products from potato starch hydrolysis in $\mathrm{LiBr}$ AMSH. Reaction conditions: $0.1 \mathrm{~g}$ of starch in 3.64 $\mathrm{ml}$ of $\mathrm{LiBr}$ AMSH, $\mathrm{T}=60^{\circ} \mathrm{C}$.

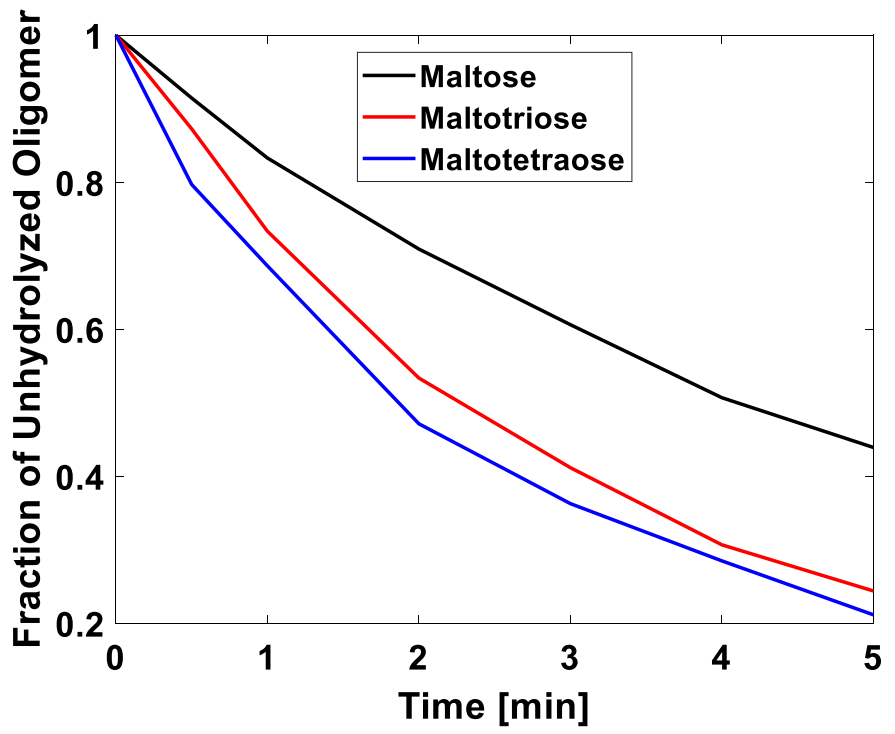

Figure A.7: Hydrolysis profile of oligosaccharides with different chain length. Reaction conditions: oligosaccharides in $3.64 \mathrm{ml}$ of $\mathrm{LiBr} \mathrm{AMSH} . \mathrm{T}=60{ }^{\circ} \mathrm{C}$. 


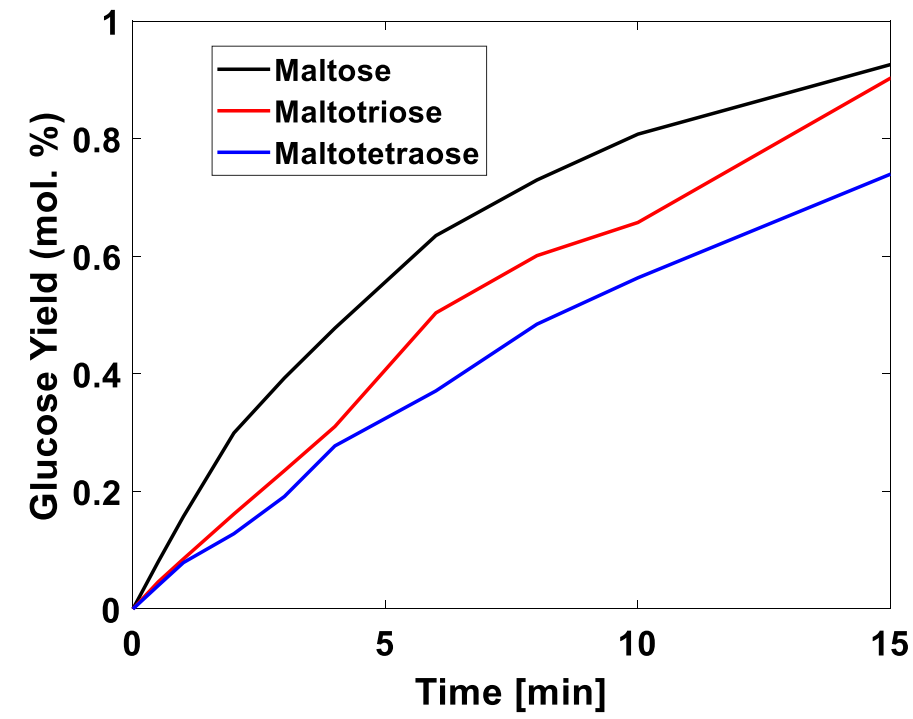

Figure A.8: Comparison of glucose yield profile from oligosaccharides with different chain length. Reaction conditions: oligosaccharides in $3.64 \mathrm{ml}$ of $\mathrm{LiBr}$ AMSH. $\mathrm{T}=60$ ${ }^{\circ} \mathrm{C}$.

\section{Matlab Code for hydrolysis simulation model}

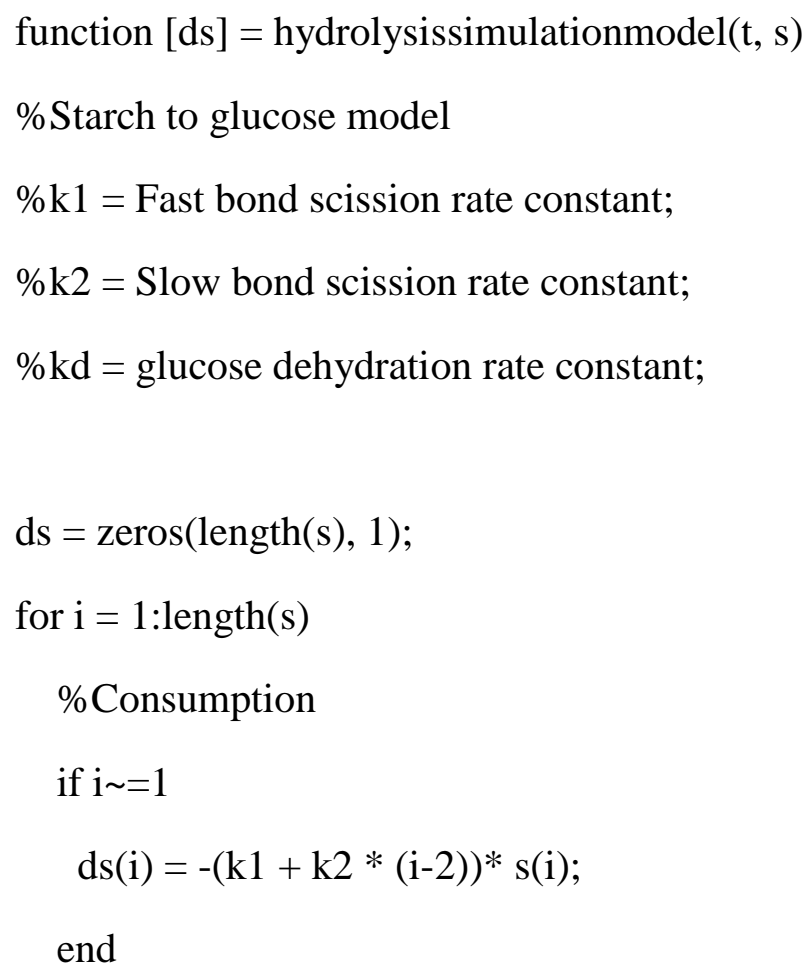




\section{\%Production}

for $\mathrm{j}=\mathrm{i}+1$ :length(s)

\%If product is glucose

if $i==1$

$\%$ And reactant is maltose

if $j==2$

\%Only k1 bond exists

$\mathrm{ds}(\mathrm{i})=\mathrm{ds}(\mathrm{i})+2 * \mathrm{k} 1 * \mathrm{~s}(\mathrm{j})$;

else

$\%$ Bond could be at either end

$$
\mathrm{ds}(\mathrm{i})=\mathrm{ds}(\mathrm{i})+(\mathrm{k} 1+\mathrm{k} 2) * \mathrm{~s}(\mathrm{j}) ;
$$

end

\%Product is larger than glucose

else

$\%$ Reactant is twice the length

if $j / i==2$

$\%$ Breaking reactant directly in half

$\mathrm{ds}(\mathrm{i})=\mathrm{ds}(\mathrm{i})+2 * \mathrm{k} 2 * \mathrm{~s}(\mathrm{j})$;

elseif $i==j-1$

$\%$ Breaking bond at end

$\mathrm{ds}(\mathrm{i})=\mathrm{ds}(\mathrm{i})+(\mathrm{k} 1+\mathrm{k} 2) * \mathrm{~s}(\mathrm{j})$;

else

\% Breaking bond somewhere in middle 


$$
\mathrm{ds}(\mathrm{i})=\mathrm{ds}(\mathrm{i})+2 * \mathrm{k} 2 * \mathrm{~s}(\mathrm{j})
$$

end

end

end

end

$\mathrm{ds}(1)=\mathrm{ds}(1)-\mathrm{kd}^{*} \mathrm{~s}(1)$

\section{Procedure to developing equations for the hydrolysis simulation model}

The hydrolysis rate of oligosaccharide $(\mathrm{n}>1)$ :

$\frac{d C_{n}}{d t}=-\left[k_{1}+(n-2) k_{2}\right] C_{n}$

The overall hydrolysis rate constant is the sum of all individual bond scission rates within the chain.

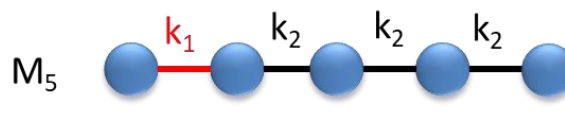

- Specific example: Maltopentaose has 5 glucan units; $\mathrm{n}=5$

- It possesses four bonds: one fast scission bond (described by $\mathrm{k}_{1}$ ) and 3 slow scission bond (described by [n-2]k $\mathrm{k}_{2}$ )

- Its rate overall rate constant of hydrolysis $=\mathrm{k}_{1}+3 \mathrm{k}_{2}$

The rate of intermediate formation (i $>1)$ :

$\frac{d c_{i}}{d t}=\left(k_{1}+k_{2}\right) C_{i+1}+\sum_{j=i+2}^{n} 2 k_{2} C_{j}$

- The first contribution accounts for an intermediate of a particular length being formed by a bond scission at either a reducing or non-reducing end, described by $\left(\mathrm{k}_{1}+\mathrm{k}_{2}\right)$.

- The second contribution accounts for an intermediate of a particular length being formed by interior bond scission at any two interior positions within the chain, described by $\left(2 \mathrm{k}_{2}\right)$. 
The rate of glucose formation:

$\frac{d C_{g}}{d t}=2 k_{1} C_{2}+\sum_{j=3}^{n}\left(k_{1}+k_{2}\right) C_{j}-k_{d} C_{g}$

- The first contribution accounts for glucose being formed directly from a maltose molecule

- The second contribution accounts for glucose being formed by a bond scission at either a reducing or non-reducing end, described by $\left(\mathrm{k}_{1}+\mathrm{k}_{2}\right)$.

- The third contribution accounts for glucose dehydration to HMF. $n=$ number of glucan monomer units within linear polysaccharide/oligosaccharide chain

$$
\begin{aligned}
& k_{1}=\text { fast bond scission rate }\left(\mathrm{min}^{-1} \text { per bond }\right) \\
& k_{2}=\text { slow bond scission rate }\left(\mathrm{min}^{-1} \text { per bond }\right) \\
& k_{d}=\text { glucose dehydration rate }\left(\mathrm{min}^{-1}\right) \\
& c_{n}, c_{i / j}, c_{2}, c_{g}=\text { initial polysaccharideloligosaccharide, intermediate }
\end{aligned}
$$

oligosaccharide, maltose and glucose concentrations $\left(\mathrm{mol} / \mathrm{dm}^{3}\right)$

Solutions of ODEs for the series reactions involving maltotriose hydrolysis to glucose

$$
\begin{aligned}
& g[t] \rightarrow e^{-k_{d} t} C_{1}-\frac{2 e^{-k} d^{t-k_{1} t}\left(-e^{k_{d} t}+e^{k_{1} t}\right) k_{1} C_{2}}{k_{d}-k_{1}}+ \\
& \frac{e^{-k_{d} t-\left(k_{1}+k_{2}\right) t-k_{1} t}\left(k_{1}+k_{2}\right)\left(\begin{array}{c}
-k_{d} e^{k_{d} t+k_{1} t}\left(k_{1}+k_{2}\right)+k_{d} e^{\left(k_{1}+k_{2}\right) t+k_{1} t}\left(k_{1}+k_{2}\right) \\
-2 k_{d} e^{k} d^{t+\left(k_{1}+k_{2}\right) t} k_{1}+3 k_{d} e^{k} d^{t+k_{1} t} k_{1} \\
-k_{d} e^{t+k_{1} t} k_{1}+2 e^{k} d^{t+\left(k_{1}+k_{2}\right) t}\left(k_{1}+k_{2}\right) k_{1}+e^{k} d^{t+k_{1} t}\left(k_{1}+k_{2}\right) k_{1} \\
-3 e^{\left(k_{1}+k_{2}\right) t+k_{1} t}\left(k_{1}+k_{2}\right) k_{1}-3 e^{k_{d} t+k_{1} t} k_{1}{ }^{2}+3 e^{\left(k_{1}+k_{2}\right) t+k_{1} t} k_{1}{ }^{2}
\end{array}\right) C_{3}}{\left(-k_{d}+k_{1}+k_{2}\right)\left(k_{d}-k_{1}\right)\left(k_{2}\right)} \\
& M_{2}[t] \rightarrow e^{-k_{1} t} C_{2}-\frac{e^{-\left(k_{1}+k_{2}\right) t-k_{1} t}\left(-e^{\left(k_{1}+k_{2}\right) t}+e^{k_{1} t}\right)\left(k_{1}+k_{2}\right) C_{3}}{k_{2}} \\
& M_{3}[t] \rightarrow e^{-\left(k_{1}+k_{2}\right) t} C_{3}
\end{aligned}
$$

Solutions of ODEs for the series reactions involving maltose hydrolysis to glucose.

$$
\left.g(t) \rightarrow C_{1} e^{-K_{d} t}-\frac{2 e^{-k_{d} t-k_{1} t}\left(-e^{k_{d} t}+e^{k_{1} t}\right) k_{1} C_{2}}{\mathrm{k}_{d}-k_{1}}\right],
$$


$M_{2}(t) \rightarrow C_{2} e^{-k_{1} t}$

$\mathrm{M}_{3}=$ Maltotriose, $\mathrm{M}_{2}=$ Maltose, $\mathrm{g}=$ glucose, $\mathrm{k}_{1}=$ rate constant for $\mathrm{C}-\mathrm{O}$ bonds scission at non-reducing terminal; $\mathrm{k}_{2}=$ rate constant for $\mathrm{C}-\mathrm{O}$ bonds scission at reducing terminal and interior linkages, $\mathrm{k}_{\mathrm{d}}=$ glucose dehydration rate constant 